\title{
Seed Population Preconditioning and Acceleration Observed by the Parker Solar Probe
}

\author{
N. A. Schwadron ${ }^{1,2}$ (D) S. Bale ${ }^{3}$ (D) J. Bonnell ${ }^{3}$, A. Case ${ }^{4}$ (D) E. R. Christian ${ }^{5}$ (D) C. M. S. Cohen ${ }^{6}$, A. C. Cummings ${ }^{6}$ (D) A. J. Davis ${ }^{6}$, \\ T. Dudok de Wit ${ }^{7}$ (D), W. de Wet $^{1}$, M. I. Desai ${ }^{8}$ (D) C. J. Joyce ${ }^{2}$ (D), K. Goetz ${ }^{3}$, J. Giacalone ${ }^{9}$, M. Gorby ${ }^{1}$, P. Harvey ${ }^{3}$, B. Heber ${ }^{10}$ (iD), \\ M. E. Hill ${ }^{11}$ (D) M. Karavolos ${ }^{12}$, J. C. Kasper ${ }^{13}$ (D) K. Korreck ${ }^{4}$ (D) D. Larson ${ }^{3}$, R. Livi ${ }^{3}$, R. A. Leske ${ }^{6}$ (D) O. Malandraki ${ }^{12}$, \\ R. MacDowall ${ }^{5}$ (D) D. Malaspina ${ }^{14}$ (D), W. H. Matthaeus ${ }^{15}$ (D) D. J. McComas ${ }^{2}$ (D) R. L. McNutt, Jr. ${ }^{11}$, R. A. Mewaldt ${ }^{6}$, \\ D. G. Mitchell ${ }^{11}$ (D) L. Mays ${ }^{5}$, J. T. Niehof ${ }^{1}$, D. Odstrcil ${ }^{5}$, M. Pulupa ${ }^{3}$ (D) B. Poduval ${ }^{1}$, J. S. Rankin ${ }^{2}$ (D), E. C. Roelof ${ }^{11}$, \\ M. Stevens ${ }^{4}$ (D) E. C. Stone ${ }_{1}^{6}$, J. R. Szalay ${ }^{2}$ (D), M. E. Wiedenbeck ${ }^{6}$, R. Winslow ${ }^{1}$ (D), and P. Whittlesey ${ }^{3}$ \\ ${ }^{1}$ University of New Hampshire, Durham, NH 03824, USA \\ ${ }^{2}$ Department of Astrophysical Sciences, Princeton University, Princeton, NJ 08544, USA \\ ${ }^{3}$ University of California at Berkeley, Berkeley, CA 94720, USA \\ ${ }^{4}$ Harvard-Smithsonian Center for Astrophysics, Cambridge, MA 02138, USA \\ ${ }^{5}$ Goddard Space Flight Center, Greenbelt, MD 20771, USA \\ ${ }^{6}$ California Institute of Technology, Pasadena, CA 91125, USA \\ ${ }^{7}$ LPC2E, CNRS and University of Orléans, Orléans, France \\ ${ }^{8}$ University of Texas at San Antonio, San Antonio, TX 78249, USA \\ ${ }^{9}$ University of Arizona, Tucson, AZ 85721, USA \\ ${ }^{10}$ Christian-Albrechts-University of Kiel, Kiel, D-24118, Germany \\ ${ }^{11}$ Applied Physics Laboratory, Laurel, MD 20723, USA \\ ${ }^{12}$ Institute for Astronomy, Astrophysics, Space Applications and Remote Sensing of the National Observatory of Athens, Vas. Pavlou and I. Metaxa, 15236 Penteli, \\ Greece \\ ${ }^{13}$ University of Michigan, Ann Arbor, MI 48109, USA \\ ${ }^{14}$ Laboratory for Atmospheric and Space Physics, University of Colorado, Boulder, CO 80303, USA \\ ${ }^{15}$ University of Delaware, Newark, DE 19716, USA \\ Received 2019 September 13; revised 2019 November 4; accepted 2019 November 5; published 2020 February 3
}

\begin{abstract}
A series of solar energetic particle (SEP) events was observed by the Integrated Science Investigation of the Sun (IS $\odot$ IS) on the Parker Solar Probe (PSP) during the period from 2019 April 18 through 24. The PSP spacecraft was located near 0.48 au from the Sun on Parker spiral field lines that projected out to 1 au within $\sim 25^{\circ}$ of the nearEarth spacecraft. These SEP events, though small compared to historically large SEP events, were among the largest observed thus far in the PSP mission and provide critical information about the space environment inside 1 au during SEP events. During this period, the Sun released multiple coronal mass ejections (CMEs). One of these CMEs observed was initiated on 2019 April 20 at 01:25 UTC, and the interplanetary CME (ICME) propagated out and passed over the PSP spacecraft. Observations by the Electromagnetic Fields Investigation show that the magnetic field structure was mostly radial throughout the passage of the compression region and the plasma that followed, indicating that PSP did not directly observe a flux rope internal to the ICME, consistent with the location of PSP on the ICME flank. Analysis using relativistic electrons observed near Earth by the Electron, Proton and Alpha Monitor on the Advanced Composition Explorer demonstrates the presence of electron seed populations (40-300 keV) during the events observed. The energy spectrum of the IS $\odot$ IS-observed proton seed population below $1 \mathrm{MeV}$ is close to the limit of possible stationary-state plasma distributions out of equilibrium. IS $\odot$ IS observations reveal the enhancement of seed populations during the passage of the ICME, which likely indicates a key part of the preacceleration process that occurs close to the Sun.
\end{abstract}

Unified Astronomy Thesaurus concepts: Solar wind (1534); Solar energetic particles (1491); Solar coronal mass ejections (310)

Supporting material: animation

\section{Introduction}

The NASA Parker Solar Probe (PSP) Mission provides humanity's first direct exploration of our star and its environment (Fox et al. 2016). The Integrated Science Investigation of the Sun (IS $\odot$ IS) instrument suite (McComas et al. 2016) provides comprehensive measurements of solar energetic particles (SEPs) using two Energetic Particle Instruments measuring higher (EPIHi) and lower (EPI-Lo) energy particles (McComas et al. 2016) over the range $0.02-200 \mathrm{MeV} /$ nucleon. Here, we examine the sources of this energetic particle environment and the seed populations therein, which respond dynamically to the solar wind observed by the Solar Wind Electrons Alphas and Protons Investigation (SWEAP; Kasper et al. 2016) and the magnetic field observed by the Electromagnetic Fields Investigation (FIELDS; Bale et al. 2016) locally around PSP. The global context for the solar wind's surrounding density structures is observed by the Wide Field Imager for Solar Probe Plus (WISPR) (Vourlidas et al. 2016), though WISPR are observations not reported in this paper.

We use SEP events observed by IS $\odot$ IS to examine the period from 2019 April 18 to 24 when two active regions near the Sun's equator became highly active, releasing numerous flares and coronal mass ejections (CMEs). We describe the SEP events observed by IS $\odot$ IS that occurred over this period. These events are the largest SEP events so far observed by PSP (McComas et al. 2019) and demonstrate the complex interplay between flares, seed populations, and CMEs in the acceleration of energetic particles near the Sun. 


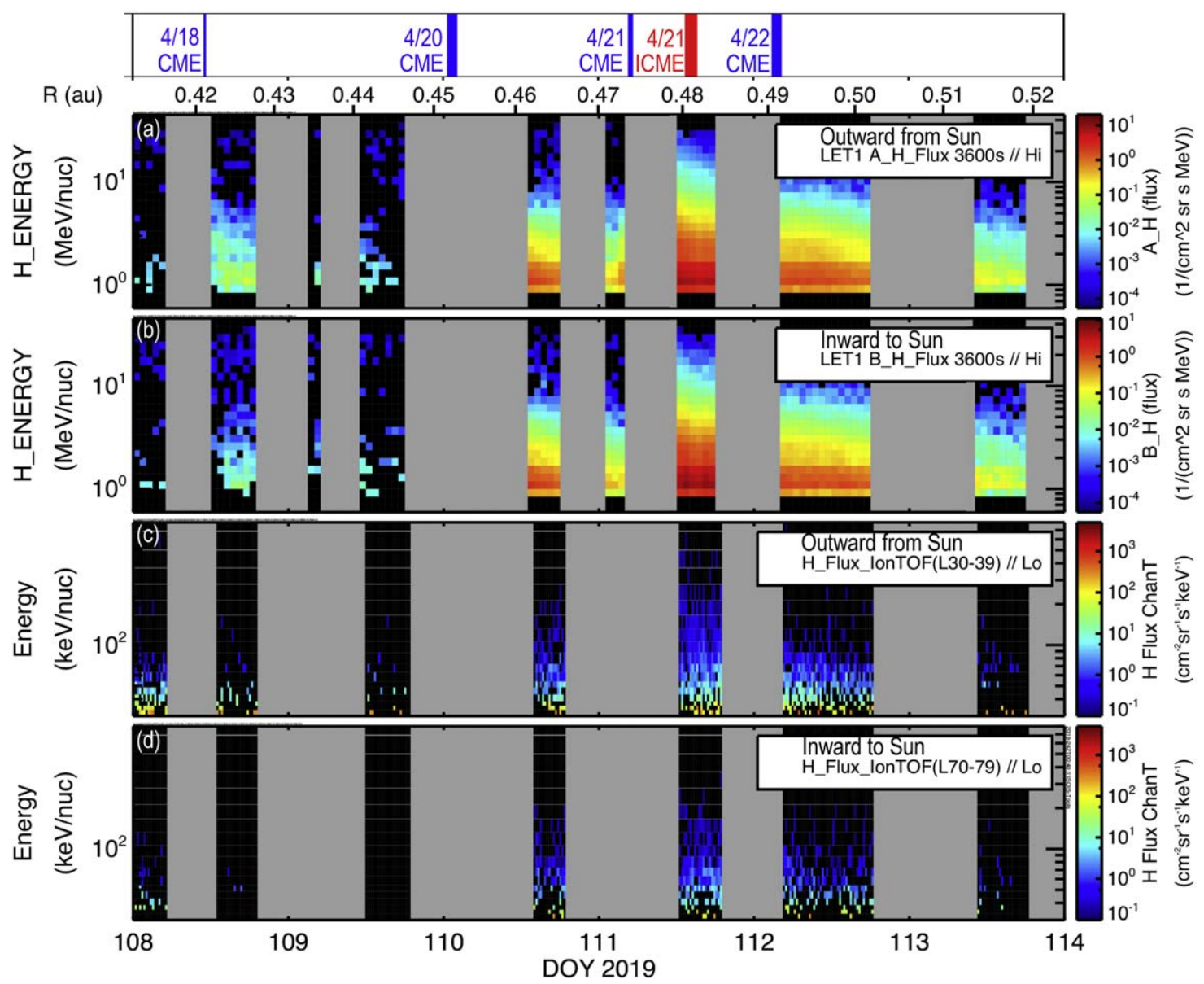

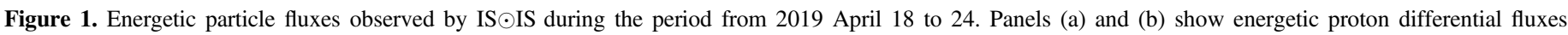

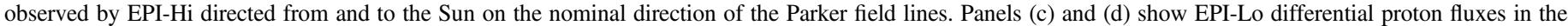

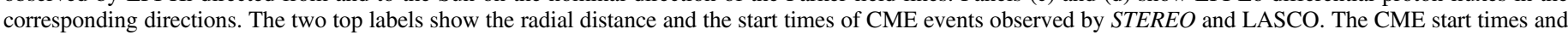

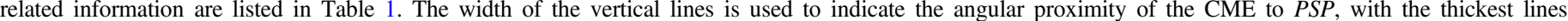

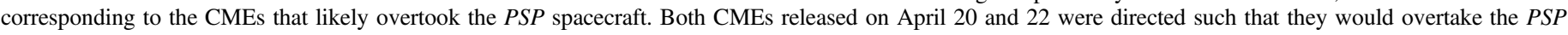

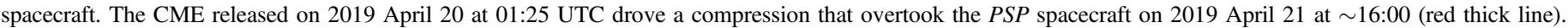

The paper is organized as follows. In Section 2, we provide a detailed analysis of the energetic particle events observed by IS $\odot$ IS from 2019 April 18 to 24 . We then assess seed populations and accelerated particles observed at PSP based on measurements of nearly scatter-free electrons in Section 3. Type III radio bursts are associated with CMEs observed over this period. We show results of a CME model in Section 4 and characterize the compression of energetic particle seed populations during the passage of an interplanetary CME (ICME) over PSP. In Section 5, we summarize key results and present our major conclusions.

\section{SEPs Observed by IS $\odot$ IS from 2019 April 18 to 24}

The energetic particle fluxes observed from 2019 April 18 (day of year, DOY 108) to 24 (DOY 114) are shown in Figure 1. The energetic fluxes in the direction outward from the Sun along a nominal Parker spiral (for a $400 \mathrm{~km} \mathrm{~s}^{-1}$ solar wind speed) are shown in panel (a) for EPI-Hi and (c) for EPI-Lo. Inward fluxes along the nominal Parker spiral are shown in panel (b) for EPI$\mathrm{Hi}$ and (d) for EPI-Lo. Observed distributions early in the event development (from DOY 108 through 109.8) show larger anisotropies. These distributions become increasingly isotropic as the associated SEP events progress after DOY 110.5.
PSP instruments were operational only intermittently during the period studied. Satellite contacts including high-speed data transfers occurred throughout the period. Instruments were powered off during these periods.

The ion spectra averaged before (April 20, 14:00-18:00), during (April 21, 12:00-18:00), and after (April 22, 04:00-18:00) the ICME passage are shown in Figure 2. We note that the spectra are all very similar, but the differential energy spectrum observed during the ICME passage is almost uniformly enhanced relative to the differential spectra observed before and after the ICME passage. In Section 4, we discuss modeling of compression and acceleration of the seed populations swept up into the compression driven by an ICME observed by PSP on 2019 April 21.

Four CMEs were released from the Sun on April 18, 20, 21, and 22. These events were identified using the Space Weather Database Of Notifications, Knowledge, Information (DONKI) provided by the Community Coordinated Modeling Center (CCMC; https://ccmc.gsfc.nasa.gov). Table 1 provides details on each of these events, including the start time, direction, PSP location, initial speed, and width of the CME. The vertical lines in the top panel of Figure 1 identify these CME release times, and the width of the vertical lines are used to indicate the angular proximity of the CME to $P S P$, with the thickest lines corresponding to CMEs that overtook the PSP spacecraft. In 


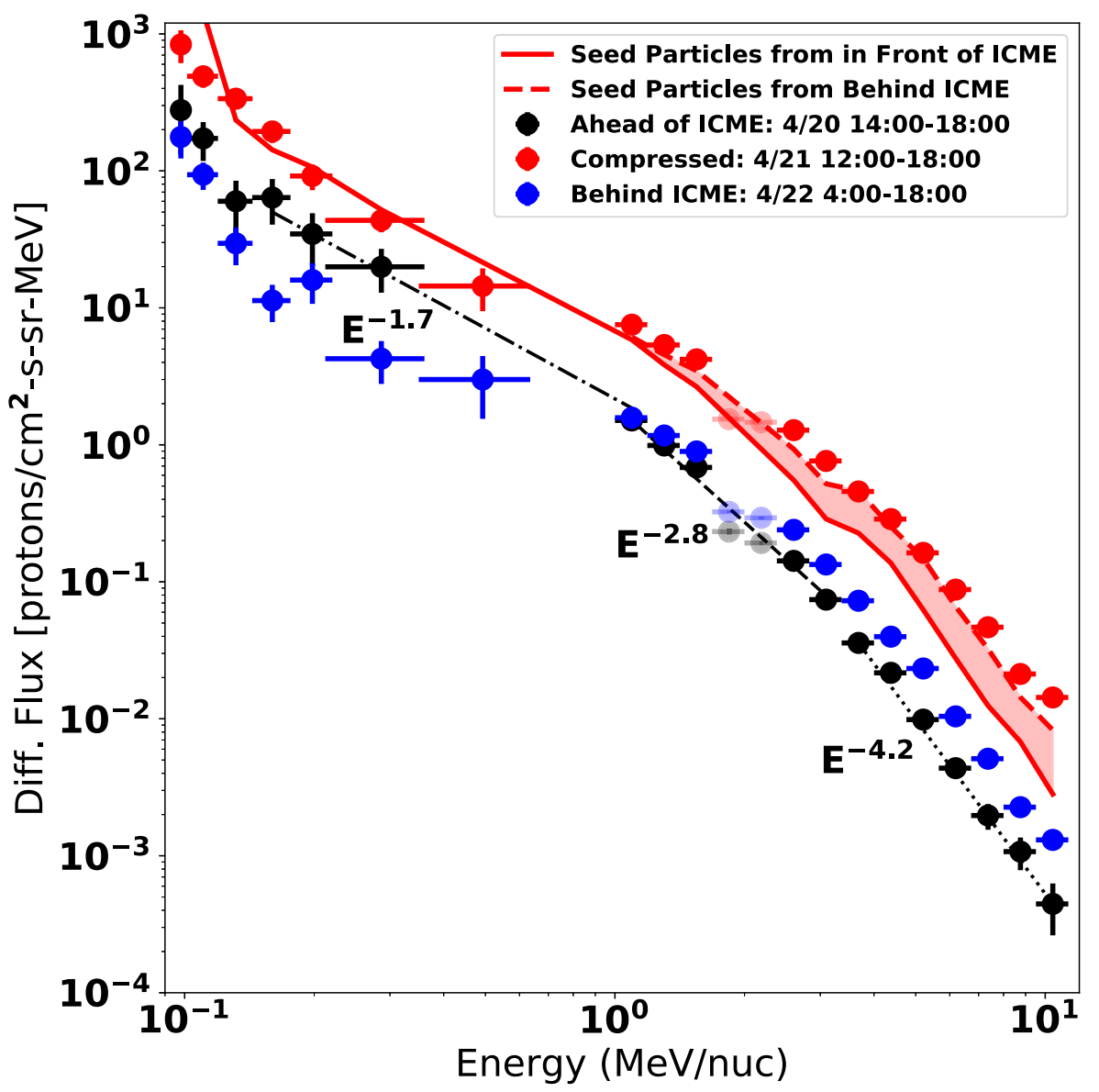

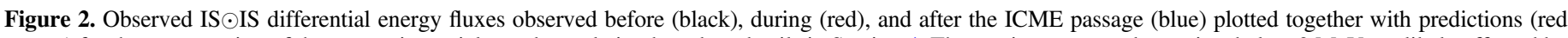

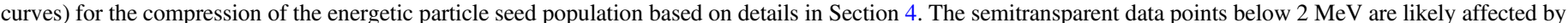

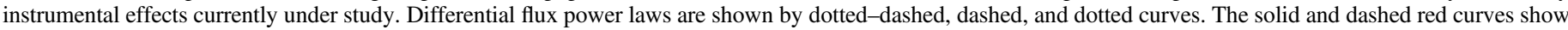

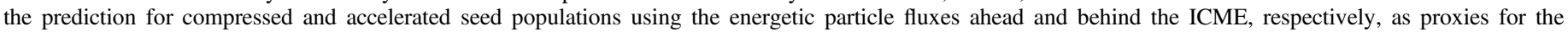
uncompressed seed population.

particular, both CMEs released on April 20 and 22, respectively, propagated in a direction such that they would overtake the PSP spacecraft.

The CME released on April 20 overtook the PSP spacecraft on April 21 near 16:00 UTC when the IS $\odot$ IS instruments, solar wind instruments (SWEAP), and magnetic field instruments (FIELDS) were powered on. Figure 3 shows the energetic particle data from EPI-Hi and EPI-Lo plotted together with plasma and field data on PSP.

Using the time difference between the $\mathrm{CME}$ release on April 20 and the observation at PSP on April 21, we infer an average ICME speed of $\sim 515 \mathrm{~km} \mathrm{~s}^{-1}$. This average propagation speed is larger than the speed of $387 \mathrm{~km} \mathrm{~s}^{-1}$ inferred from observations of CMEs near the Sun listed in the DONKI database. However, CME measurement near the Sun remains somewhat subjective (Webb \& Howard 2012) , and coronagraphs identify the propagation of the core CME that drives the plasma. The identification of shocks or compressions in front of the core CME (often in the form of a flux rope) in white-light coronagraph images has been very difficult (e.g., Vourlidas et al. 2003; Ontiveros \& Vourlidas 2009; Vourlidas \& Ontiveros 2009). Therefore, the larger average propagation speed deduced from the compression that leads the CME compared to the speed derived from coronagraph images is expected. The arrival time of 16:00 UTC is similar to the arrival time at PSP of 19:09 UTC predicted from the WangSheeley-Arge (WSA)-ENLIL+Cone Model (https://kauai. ccmc.gsfc.nasa.gov/DONKI/view/WSA-ENLIL/14640/1), which is detailed in Section 4 and Appendix C.

Shown in Figure 4 is an expanded view of the ICME passage. The compression shows the plasma speed rising from $V \sim 300 \mathrm{~km} \mathrm{~s}^{-1}$ to $V \sim 380 \mathrm{~km} \mathrm{~s}^{-1}$. The SWEAP analysis during this period was a particular challenge because the solar wind signature in SWEAP was difficult to identify unambiguously. After averaging the more than two-hour time period used for the second SWEAP data point centered at about 16:00 UTC on April 21, we were able to identify a solar wind signature. A gradient in the radial solar wind speed is observed along with a rise in the thermal speed, suggesting that a compressional plasma structure passed by the spacecraft. However, the lack of time resolution makes it difficult to determine whether the structure was a shock or a compression. The final speed of the structure and the associated plasma density are also undetermined. The average propagation speed of $515 \mathrm{~km} \mathrm{~s}^{-1}$ was deduced from the distance to PSP divided by the propagation time of the CME to the PSP spacecraft. The propagation time was from the CME initiation observed in coronagraph images to the arrival time at $P S P$. The average $515 \mathrm{~km} \mathrm{~s}^{-1}$ CME speed and the slow wind speed of $\sim 300 \mathrm{~km} \mathrm{~s}^{-1}$ in front of the ICME suggest that the compression ratio was of order $V_{f} / V_{s} \approx 1.7$, 


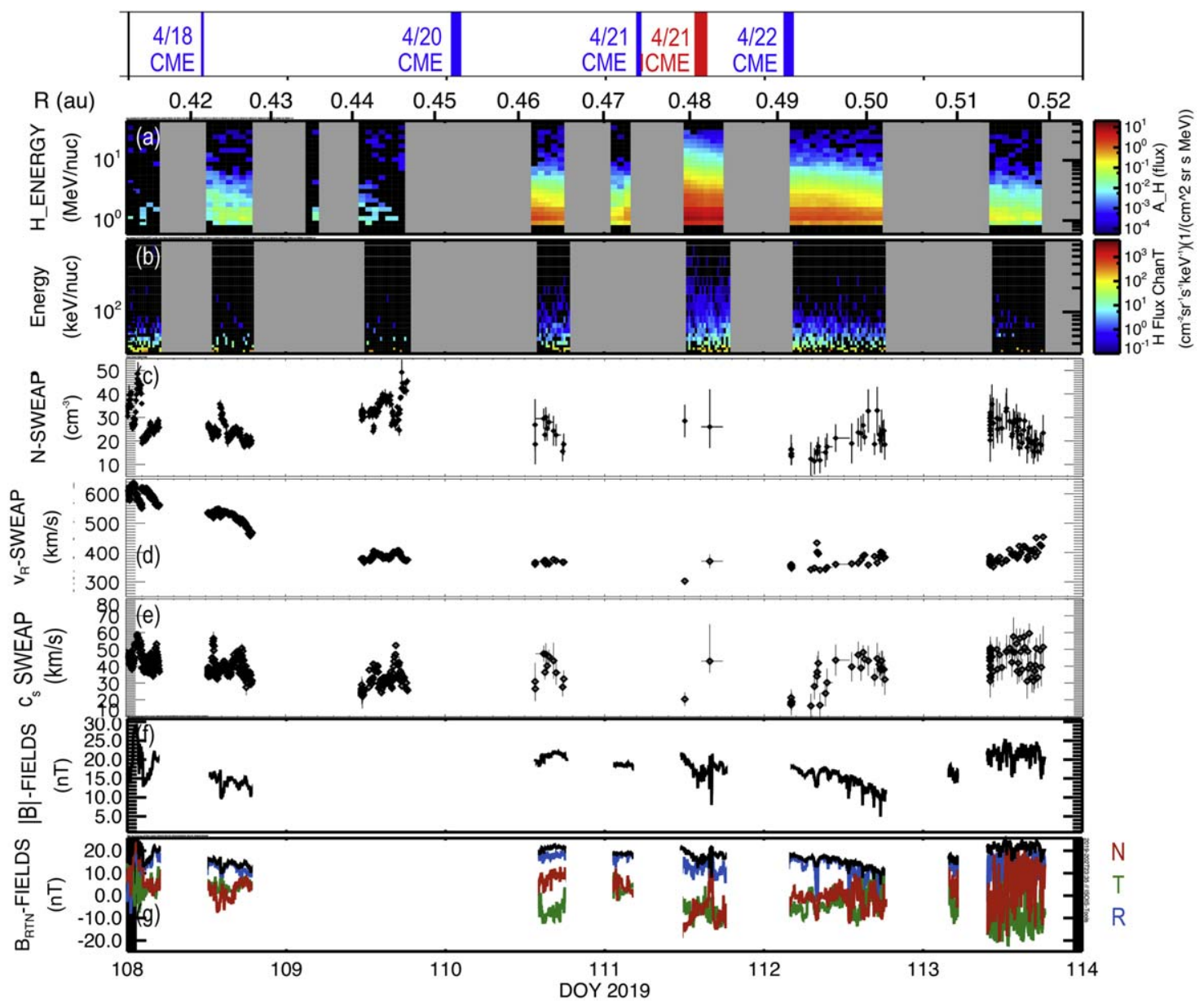

Figure 3. Energetic particle fluxes (outward along the Parker spiral) observed by IS $\odot$ IS are shown together with SWEAP plasma and FIELDS observations during the period from 2019 April 18 to 24. Panels (a) and (b) show the energetic proton differential fluxes observed by EPI-Hi and EPI-Lo, respectively, in directions outward away from the Sun along the nominal Parker spiral magnetic field. Panels (c)-(g) show the solar wind density, radial wind speed, thermal speed, magnetic field strength (black curve in panels (f) and (g)), and RTN field components. Here, RTN refers to the radial ( $\boldsymbol{r}$; blue curve), tangential $\left(\boldsymbol{t} \propto \boldsymbol{\Omega}_{\odot} \times \boldsymbol{r}\right.$; green curve), and normal $\left(\boldsymbol{n}=\boldsymbol{r} \times \boldsymbol{t}\right.$; red curve) orthonormal components with $\Omega_{\odot}$ defined as the spin axis of the Sun (in J2000). The two top labels show the radial distance and the start times of CME events observed by STEREO and LASCO.

Table 1

CMEs Released from 2019 April 18 to $24^{\mathrm{a}}$

\begin{tabular}{|c|c|c|c|c|c|c|}
\hline Date & $\begin{array}{c}\text { Time } \\
Z\end{array}$ & $\mathrm{CME}^{\mathrm{a}}$ & $P S P^{\mathrm{a}}$ & $\begin{array}{c}\text { Speed } \\
\left(\mathrm{km} \mathrm{s}^{-1}\right)\end{array}$ & $\begin{array}{l}\text { Width } \\
\left(^{\circ}\right)\end{array}$ & Type III \\
\hline 2019 Apr 18 & 11:09 & $\left(-149^{\circ}, 1^{\circ}\right)^{b}$ & $\left(57^{\circ}, 1^{\circ} .5\right)$ & 428 & 44 & STEREO-A \\
\hline 2019 Apr 20 & $01: 25$ & $\left(90^{\circ}, 2^{\circ}\right)^{c}$ & $\left(60^{\circ}, 1.8\right)$ & 387 & 60 & WIND \\
\hline 2019 Apr 22 & $03: 36$ & $\left(87^{\circ}, 9^{\circ}\right)^{\mathrm{e}}$ & $\left(63^{\circ}, 2^{\circ}\right)$ & 434 & 52 & WIND $^{\mathrm{f}}$ \\
\hline
\end{tabular}

Notes.

${ }^{\text {a }}$ Longitude and latitude in HEEQ coordinates.

b The source observed by STEREO A EUVI $195 \AA$ A began at 10:05 UTC and was characterized by dimming and opening field lines along with a post-eruptive arcade.

${ }^{c}$ The eruption from AR 12738 that caused this CME corresponded to a B8.1 flare from the active region and a filament eruption visible off the western limb that began at 00:42 UTC.

d Initial source is an eruption from AR 12738, just beyond the western limb in AIA 171 and 304, at 03:24 UTC. Later eruption is visible behind the western limb in AIA 171 and 304 at 05:00 UTC, which may have contributed to the later/brighter inner edge of the ejecta seen in the CME.

${ }^{\mathrm{e}}$ The source is an eruption in AR 12738 around 02:50 UTC. The CME is very faint, and the real-time measurements were done while there was a data outage in STA Cor2.

${ }^{\mathrm{f}}$ Very weak type III emissions were observed prior to and during the onset of the CME event on April 22.

where $V_{f}$ is the fast wind speed and $V_{s}$ is the slow wind speed. This compression ratio must be taken as a crude estimate given the lack of higher resolution plasma data. We note from the modeled plasma timeline shown in Appendix $\mathrm{C}$ that the $\mathrm{CME}$ may have accelerated as part of a larger scale stream interaction region. If this were the case, the CME compression region forms part of an even larger compression region within the solar wind. 


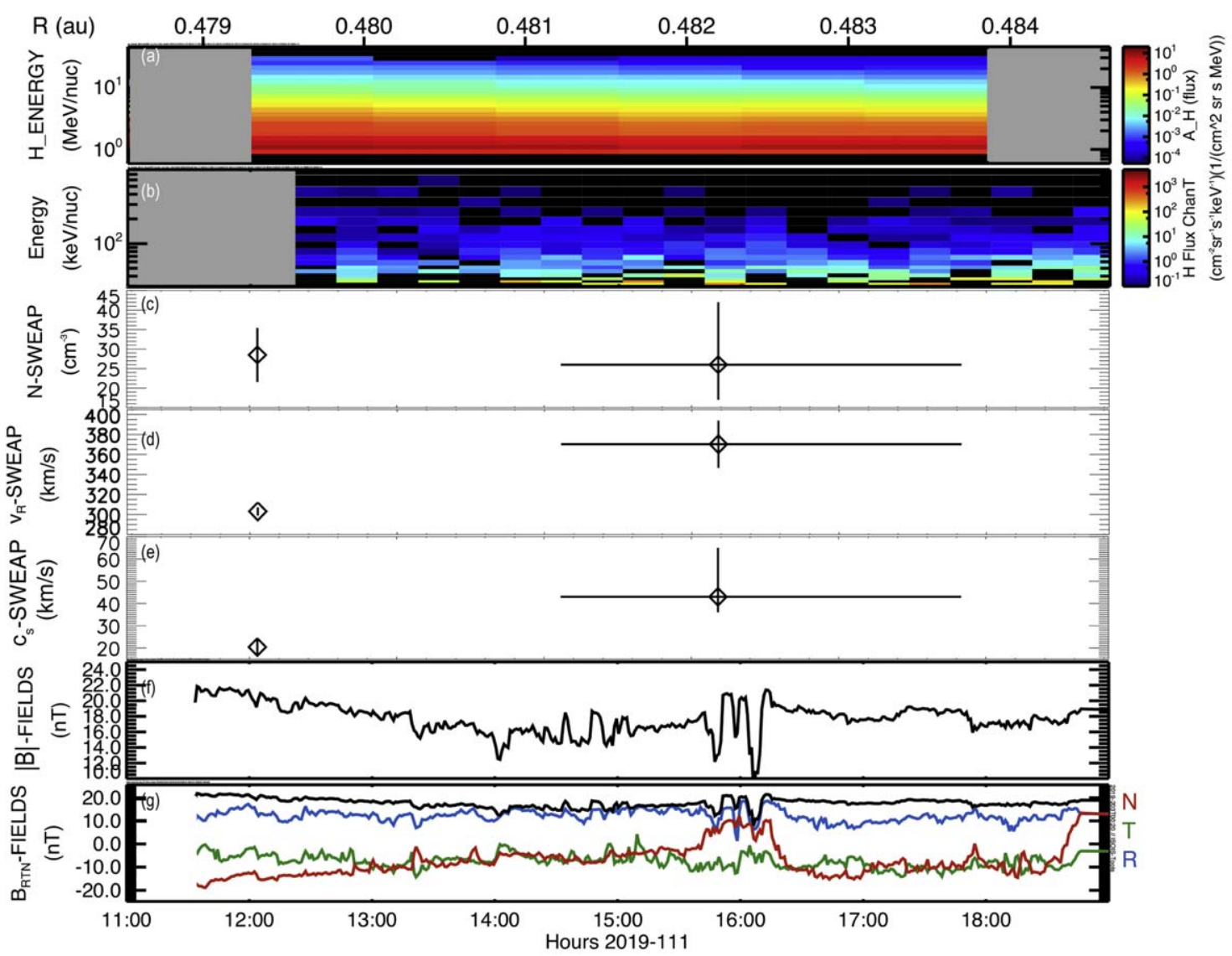

Figure 4. Energetic particle fluxes observed by IS $\odot$ IS (outward along the Parker spiral) together with plasma observations during the passage of the ICME on April 21. This ICME was associated with the CME released from the Sun on April 20. The average ICME propagation speed of $515 \mathrm{~km} \mathrm{~s}^{-1}$ was inferred from the time difference between the compression passage at PSP and the CME release. Panels (a) and (b) show the energetic proton differential fluxes observed by EPI-Hi and EPILo in directions outward away from the Sun along the nominal Parker spiral magnetic field. Panels (c)-(g) show the solar wind density, radial wind speed, thermal speed, magnetic field strength (black curve in panels (f) and (g)), and RTN (blue, green and red curves, respectively) field components. The top label shows the radial distance of the $P S P$ spacecraft.

It is possible that the compressed plasma within the ICME is a remnant of an already merged structure. If this were the case, the ICME plasma may have been moving more quickly upstream, and subsequently slowed as the fast flow merged with the slower flow. Therefore, we take a compression ratio of $\sim 1.7$ as a lower limit.

As shown in the bottom panel of Figure 4, the radial magnetic field (blue curve) and the magnetic field strength (black curve) are almost equal throughout the April 21 period. The magnetic field has a large radial component throughout the ICME passage, making it unlikely that a flux rope passed over the PSP spacecraft. The largest, $\sim 55^{\circ}$, deviation of the field from radial occurs from 16:30 to 17:30 on April 21. As discussed in Section 4, modeling consistently suggests that the PSP spacecraft was overtaken by the flank of the ICME.

\section{Contemporaneous Remote and In Situ Observations}

In assessing the source of seed populations and accelerated particles observed at $P S P$, it is important to identify solar flares and type III radio bursts observed over the period studied. Energetic electrons are often nearly scatter free (e.g., Lin 1974) and provide an unambiguous identification of energetic particle seed populations. Further, the observed type III emissions over this period provide remote association with energetic particles released near the CME ejection.

The PSP spacecraft was east of Earth in the heliocentric frame (i.e., ahead and upstream of Earth in its orbit) on Parker spiral field lines less than $25^{\circ}$ from the near-Earth spacecraft. Figure 5 shows the $P S P$ orbit relative to Earth and other planets within the inner heliosphere, and relative to the STEREO A spacecraft. This configuration proves to be important because the Advanced Composition Explorer (ACE) spacecraft located at the Lagrangian L1 point was close to being magnetically connected to PSP. As such, we use observations from $A C E$ of energetic electrons as indicators of seed populations.

Figure 6, panel (c) shows an overview of the 20 minute and spin-averaged differential intensities of four electron channels (DE1-DE4: 38-315) as measured by the B detector head of the CA60 telescope of the Electron, Proton and Alpha Monitor (EPAM) experiment on ACE. Details regarding the instrument and observations over the period studied are provided bin Appendix A. Panel (d) shows the 20 minute and spin-averaged differential intensities of the 1.1-4.9 MeV energetic ions measured by the LEMS120 telescope of the EPAM experiment for the same interval.

On April 20 and 21, two near-relativistic prompt electron events were observed (E1 and E2). The electron event on April 21 (E2) is more intense and extends to a higher energy range, up 


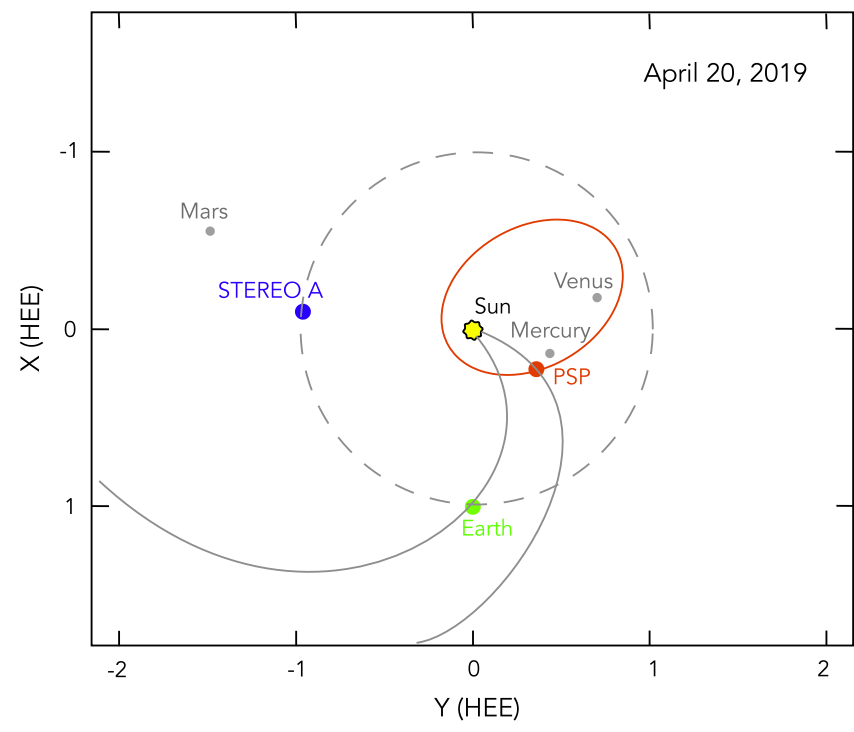

Figure 5. Illustration of the position of PSP in the ecliptic plane on 2019 April 20 relative to Earth and other planets within the inner heliosphere and STEREO $A$. The red curve shows the second orbit of PSP about the Sun, and the solid gray curves show nominal $\left(400 \mathrm{~km} \mathrm{~s}^{-1}\right)$ Parker spiral magnetic fields lines connected to $P S P$ and to Earth. The dashed gray curve represents the 1 au circle in the ecliptic plane. The coordinate system used here is Heliocentric Earth Ecliptic (HEE; Russell 1971; Hapgood 1992; Fränz \& Harper 2002).

to $315 \mathrm{keV}$. An electron event is observed superposed during the decay phase of the first event of this period. The electron events exhibit typical rise time to maximum of a few tens of minutes, a long smooth decay (Lin 1970, 1974), and beam-like pitch-angle distributions, as detailed in Appendix A. In these prompt electron events, particles accelerated in a magnetically wellconnected solar source region arrive abruptly at the spacecraft (e.g., Reames 1999; Malandraki et al. 2002). Two, relatively weak, proton intensity enhancements (Figure 6, panel (d)) are observed in association with these electron events.

Appendix A discusses observed the electron pitch-angle distributions used to estimate the event onset times at the Sun: E1 at 00:44 UTC on April 20, roughly 40 minutes prior to the corresponding CME release time listed in Table 1; and E2 at 04:47 UTC on 2019 April 21, which is 13 minutes prior to the CME release time listed in Table 1.

Both events on April 20 and 21 showed strong enhancements in ${ }^{3} \mathrm{He}$ (Wiedenbeck 2020) observed by IS $\odot$ IS. These strong enhancements definitively support the concept that energetic particle seed populations contained flare-accelerated material (Mason et al. 1986, 2002; Reames 1999; Desai et al. 2003; Bučík et al. 2015, 2016).

The CMEs initiated on April 18, 20, and 21 (Table 1) were associated with the type III bursts observed by the STEREO A Radio and Plasma Wave Investigation (WAVES) on April 18, and by the Wind Radio and Plasma Wave Investigation (WAVES) on April 20 and 21. ${ }^{16}$ There were only weak type III emissions observed by Wind/WAVES on April 22 prior to and during the CME initiation. Type III bursts start at around $10 \mathrm{MHz}$ and then progress to lower frequencies with time. These bursts are delayed with respect to the associated flare and last $\sim 20$ minutes on average. Type III bursts are associated with CMEs and typically SEPs (Cane et al. 2002; MacDowall et al. 2003).

\footnotetext{
${ }^{16}$ Browse data for these experiments are available at the Goddard Space flight Center STEREO Science Center, https://stereo.gsfc.nasa.gov/browse/.
}

However, Gopalswamy \& Mäkelä (2010) found that a type III burst does not always signify the presence of solar energetic protons.

\section{Modeling of the April 20-21 SEP Event at PSP}

The energetic particle events studied were associated with CMEs, suggesting a relationship with a solar active region. The $P S P$ spacecraft was at heliocentric distances ranging from 0.46 to 0.49 au during the April 20-22 time period. The SEP events detected by PSP were mapped along the Parker spiral back to the Sun using the Current Sheet Source Surface (CSSS) Model, detailed in Appendix B.

In Figure 7, the black dotted line represents the solar equator, and the symbols (triangles) indicate the footpoint locations on the source surface at $15 R_{s}$ in the corona mapped back on April 20, 21, and 22. The filled circles show the respective photospheric footpoints mapped back along the open magnetic field lines. Note that the photospheric footpoints lie close to the active region (AR 12738) boundaries.

Figures 8 and 9 show the propagation of the CME released on April 20 from the Sun using the Enlil model (Odstrcil 2003) initialized using CME parameters from the CCMC's DONKI database. The location of PSP is on the flank of the CME, which is consistent with plasma compression during the passage of the ICME without the accompanying signature of a flux rope. Further results from Enlil modeling are detailed in Appendix $\mathrm{C}$ and includes a movie of the CME; see Figure 13.

The compression ratio, estimated to be $r_{c}=1.7$, is difficult to determine because SWEAP observations were incomplete during the ICME passage, and the plasma speed and density were likely time variable, as suggested by the results of the Enlil model (Figures 8 and 9). This compression ratio is similar to that found from the Enlil simulation.

The ${ }^{3} \mathrm{He}$ enhancements observed during this period (Wiedenbeck 2020) confirm that solar flares are responsible for producing at least part of the energetic particle seed population observed. Appendix D considers the situation where an energetic particle population is swept up, compressed, and accelerated with the solar wind plasma in front of the ICME, as depicted in Figure 10.

Appendix D shows that if the width of the compression is wider than the diffusion region, particle distributions are convected through the compression and accelerated by the solar wind speed gradient. As a result, the distribution function in the compression is given by $f_{c}=r_{c}^{\gamma / 3} \tilde{f}_{0}$, where $r_{c}$ is the compression ratio $\left(r_{c}=1.7\right)$ and $\gamma$ is the power-law index of the energetic particle distribution $\tilde{f}_{0}$ in the faster wind behind the compression such that $\tilde{f}_{0} \propto p^{-\gamma}$. The criterion required for this form of acceleration is that the scattering mean free path $\lambda$ must be sufficiently small compared to the width, $\delta x$, of the speed gradient to restrict diffusion upstream. Therefore, $\lambda<3 u \delta x / v$, where $u$ and $v$ are the solar wind and particle speeds, respectively. We estimate the compression region width of $\sim 0.07$ au based on an average convection speed of $400 \mathrm{~km} \mathrm{~s}^{-1}$ and the time period of $\sim 7 \mathrm{hr}$ over which the enhanced energetic particle fluxes are observed (see Figure 4). For these parameters, the mean free path of a $1 \mathrm{MeV}$ proton would have to be less than 0.006 au or $\sim 1.3 R_{s}$ to restrict upstream diffusion.

For reference, we show the modeled time profile of the event at PSP in Appendix C. The modeled compression region is more than 0.25 au in scale, which is larger than the inference using IS $\odot$ IS data. A significant portion of the compression region 

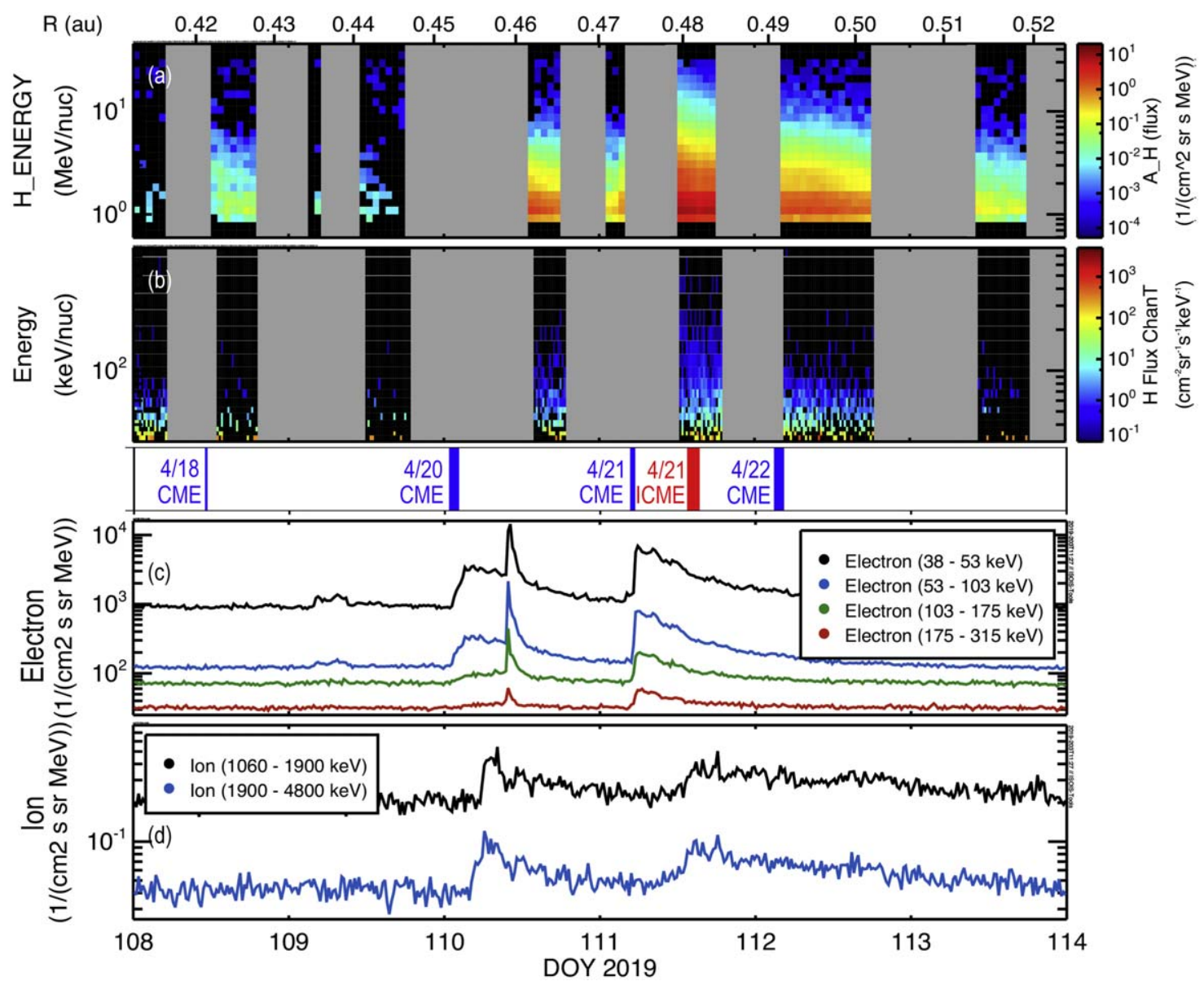

Figure 6. Energetic particle fluxes (panels (a) and (b), outward along the Parker spiral) together with spin and 20 minute averaged intensities of 38-315 keV electrons vs. time, observed with the ACE/EPAM experiment in the interval 2019 April 18-24 (panel (c)). 1.1-4.8 MeV spin and 20 minute averaged ion intensities observed with the LEMS120 detector of the ACE/EPAM experiment (panel (d)). The middle label shows CMEs identified in Table 1 and the top label shows the radial distance of the PSP spacecraft.

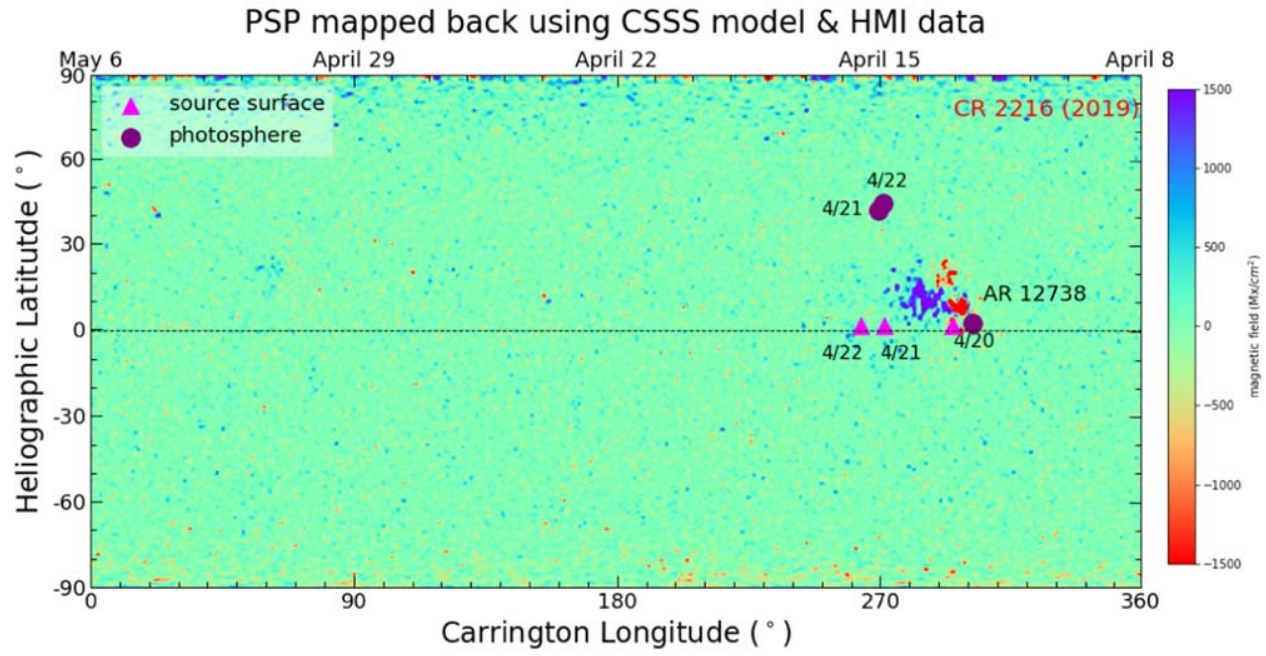

Figure 7. Source surface model to map magnetic fields from the PSP location to source regions at the Sun. The triangles are the angular positions of $P S P$ mapped back to the source surface at $15 R_{s}$, and the circles show the corresponding footpoints at the photosphere on April 20,21, and 22. These footpoints were back-mapped using solar wind speeds of $300,350,400 \mathrm{~km} \mathrm{~s}^{-1}$, respectively. Footpoints are overplotted on the HMI synoptic map for Carrington rotation CR 2216 which includes the SEP event observed by PSP during April 20-22.

appears to be missed when the instruments were powered off over satellite contacts and high-speed data transfers. This potentially larger size of the compression region would slightly relax the limit on the scattering mean free path, $\lambda<0.02$ au. More importantly, the model results indicate that the ICME may be in the process of being enveloped in a stream interaction region. 
(a)

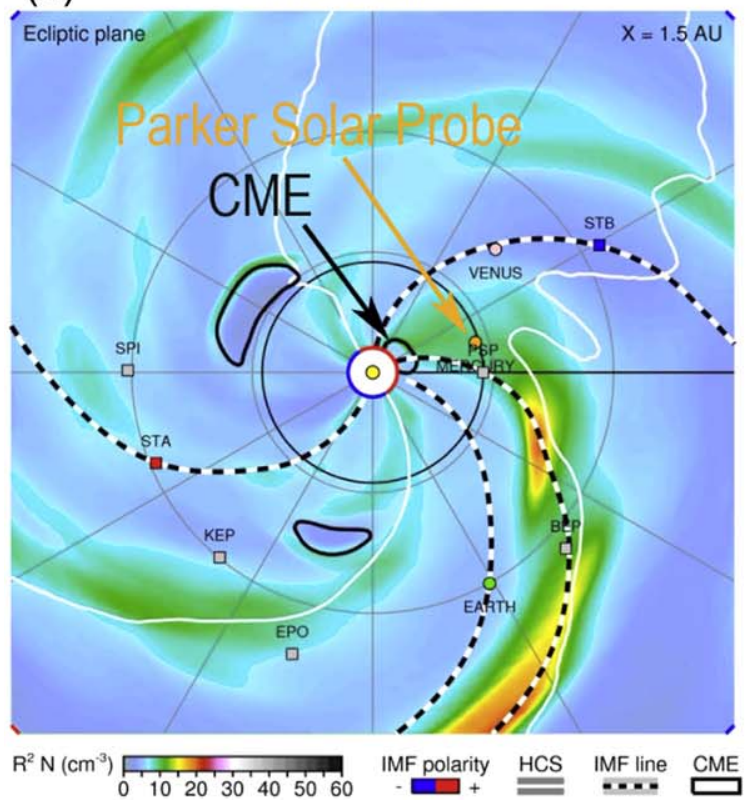

(b)

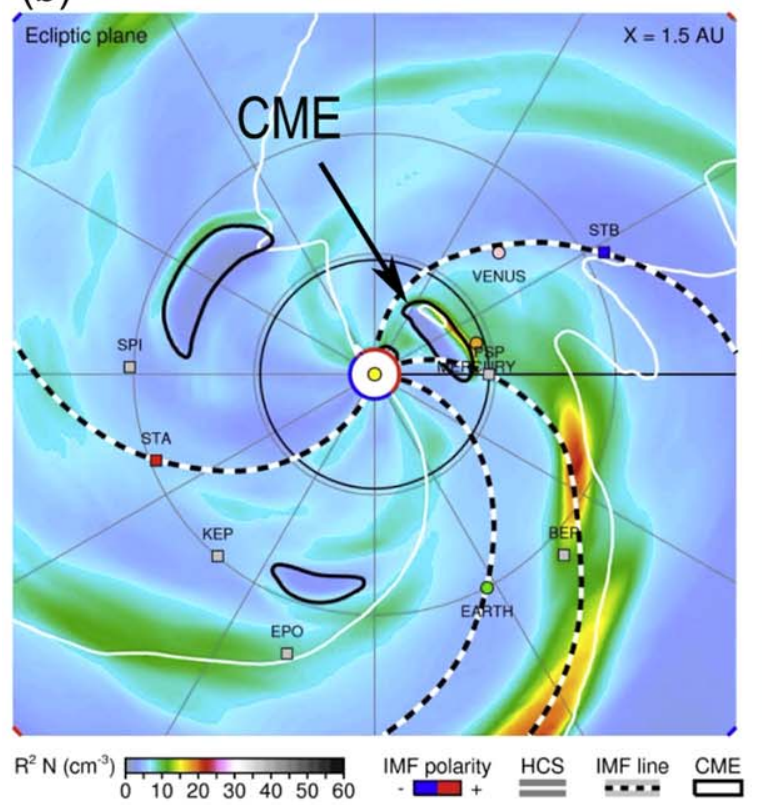

2019-04-20T19:02

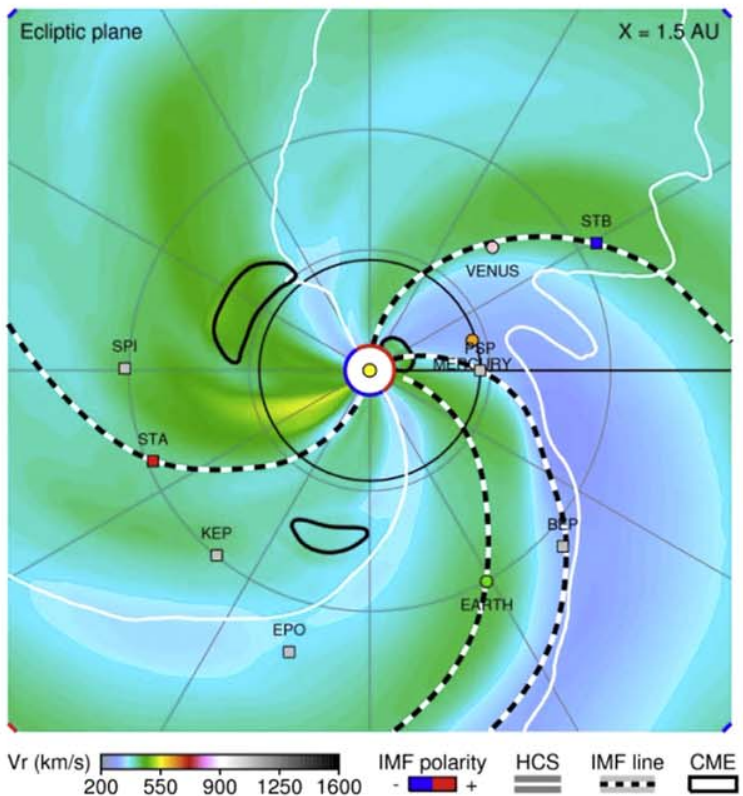

2019-04-21T16:02

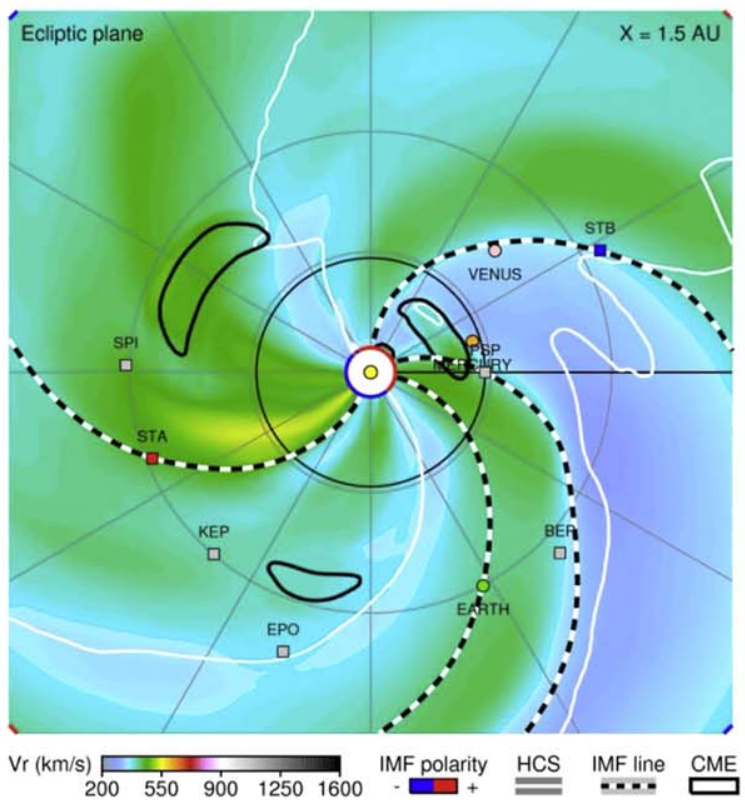

Figure 8. Snapshots showing the Enlil model of the 2019 April 20 CME released at 01:25 UTC and propagating out to PSP. Panels (a)-(b) show the evolution of the CME through the inner heliosphere at different stages of the CME's propagation to the PSP. Left panels show modeled densities and right panels show the speed structure from the 3D model.

Figure 1 shows the energetic proton differential fluxes observed by EPI-Hi and EPI-Lo directed from and to the Sun on the nominal direction of Parker field lines. These observations show that the distributions are closest to being isotropic at the highest energies observed, and we observe an outward anisotropy in the spacecraft reference frame, which is most pronounced at the lowest energies observed. The observations are consistent with distributions that are close to being isotropic in the solar wind reference frame. Standard diffusion theory (e.g., Forman \& Gleeson 1975) specifies the energetic particle anisotropy (the Compton-Getting term) in the spacecraft reference frame, $\boldsymbol{\xi} \approx \gamma \boldsymbol{u} / \nu$. Below $1 \mathrm{MeV}$, observations within the compression indicate $\gamma \approx 5.4$. At $100 \mathrm{keV}$, the inferred anisotropy magnitude is $\sim 50 \%$, and at $1 \mathrm{MeV}$, the anisotropy magnitude drops to $16 \%$. Both the outward direction of the observed anisotropy and its magnitude appear roughly consistent with the Compton-Getting term.

In Figure 2, we show the energetic particle distributions (red curves) that result from the compressive acceleration of seed populations. For energies below $1 \mathrm{MeV}$, we use the energetic particle fluxes ahead of the CME (black data points) as a proxy for the uncompressed seed population. Above $1 \mathrm{MeV}$, the solid red curve shows the prediction using the energetic particle fluxes ahead of the ICME (black points) as the proxy for the seed population and with power-law indices $(\gamma)$ based on the two fits indicated in the figure $\left(J \propto E^{-2.8}\right.$ for 
(c)

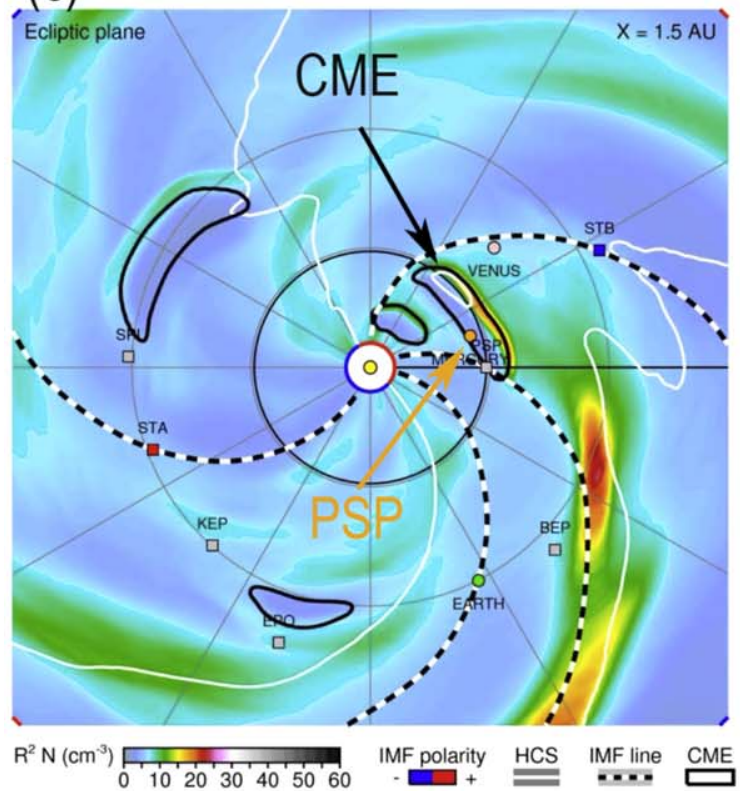

(d)

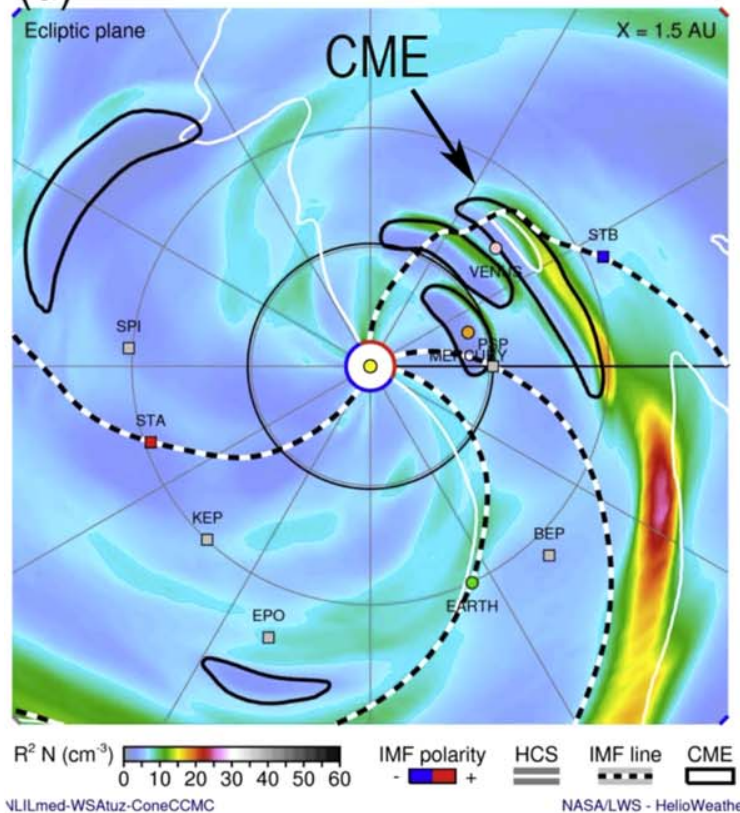

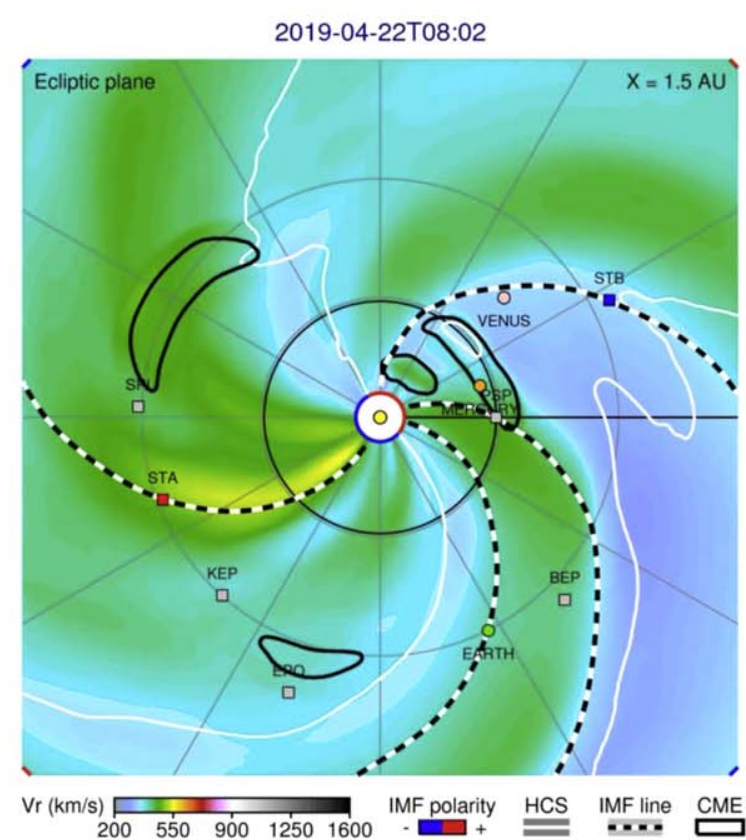

2019-04-23T22:01

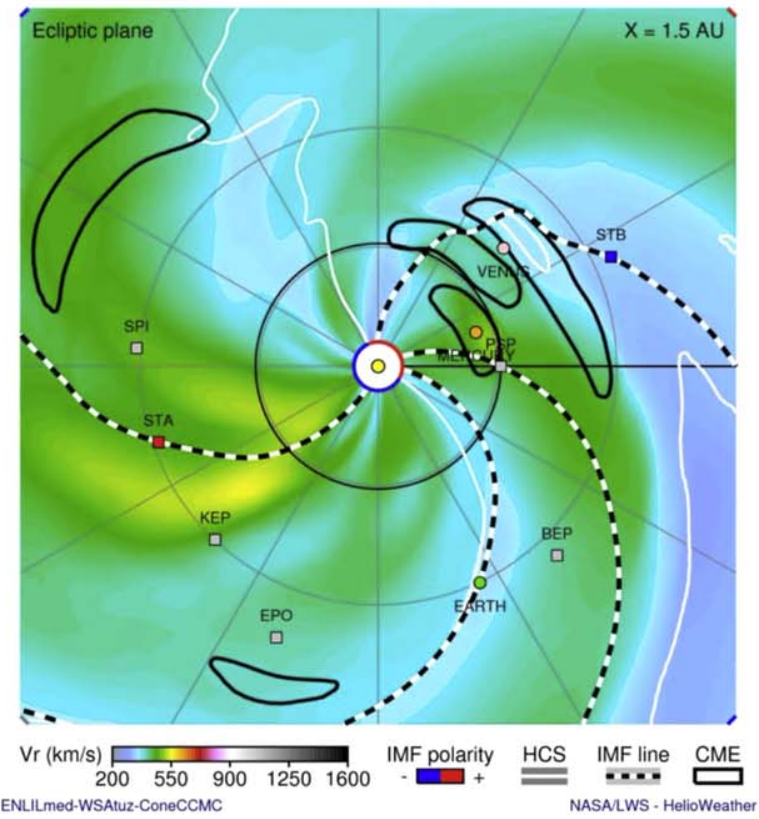

Figure 9. Enlil model snapshots similar to Figure 8 showing the stages of the CME's propagation after April 21 out to 1 au.

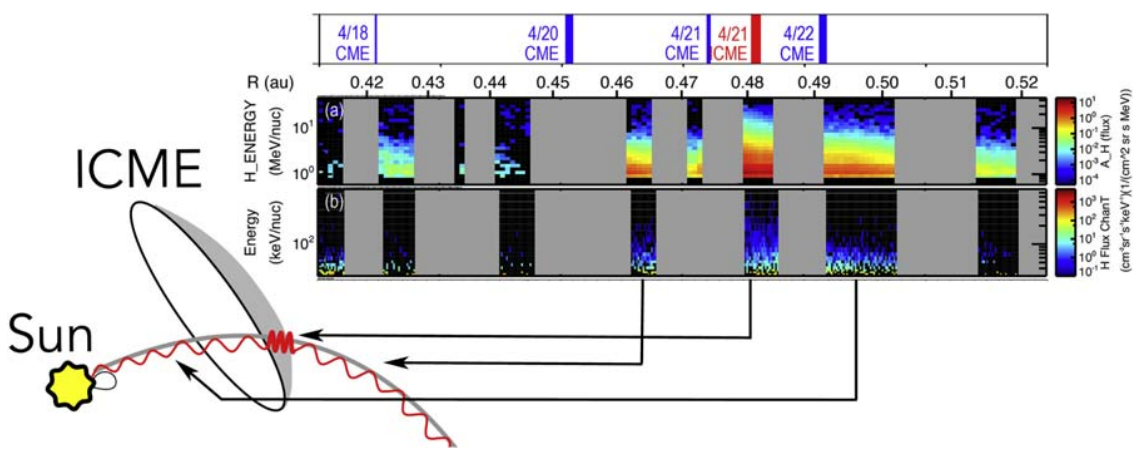

Figure 10. Schematic of the ICME, which drives a compression region (shown by gray region) ahead of the ICME. We show the fluxes observed by IS $\odot$ IS before, during, and after the ICME passage, and indicate where relative to the ICME these populations are observed. 
$1 \mathrm{MeV}<E \leqslant 3 \mathrm{MeV}$ and $J \propto E^{-4.2}$ for $E>3 \mathrm{MeV}$ ). The dashed red curve shows the prediction using the energetic particle fluxes behind the ICME (blue points) as the proxy for the seed population.

There is a fundamental change in energetic particle fluxes between energies below and above $1 \mathrm{MeV}$. The fluxes ahead of the ICME provide better proxies for the uncompressed seed population below $1 \mathrm{MeV}$, but the fluxes of seed populations were likely time variable over the period observed. For example, it is likely that additional seed populations were associated with the April 21 04:47 UTC CME. These additional fluxes may account for the change in the seed population above $1 \mathrm{MeV}$. This scenario is consistent provided that slower protons from the April 21 flare with energies $<1 \mathrm{MeV}$ did not propagate to $P S P$ during the ICME passage, whereas faster $>1 \mathrm{MeV}$ protons were capable of propagating to the spacecraft.

The propagation of seed populations following a delta function injection at the Sun creates a spatially and temporally dependent variation of the seed particle distribution function (e.g., Schwadron \& Gombosi 1994). Early in an event, particles move out from a flare at the causal limit dictated largely by the particle speed, and later in events, distributions relax into diffusive propagation. A $1 \mathrm{MeV}$ proton has a speed of $v=13.8 \times 10^{3} \mathrm{~km} \mathrm{~s}^{-1}$. We take a propagation distance of $\delta x=0.48$ au to $P S P$ and a propagation time of $\delta t=11.2 \mathrm{hr}$ from the point of energetic particle injection to the observation time at PSP. The causal limit for propagation is at a distance of $3.7 \mathrm{au}$. Because PSP is so much closer in at $0.48 \mathrm{au}$, we can safely consider distributions that evolve diffusively, with a spatial profile $f_{D} \propto \exp \left(-x^{2} /\left[4 \kappa_{\|} t\right]\right)$. We take the parallel diffusioncoefficient given by $\kappa_{\|}=\lambda v / 3$, where $\lambda$ is the scattering mean free path. We use the proxy that the characteristic diffusive propagation distance is where the spatial profile falls to a factor of 2 lower than near the source, allowing us to estimate the one unknown, the scattering mean free path, $\lambda=3 \delta x^{2} /(4 \ln (2) v \delta t) \approx 0.1 \mathrm{au}$. This estimate appears roughly consistent with scattering mean free paths observed previously over similar ranges of rigidity (Bieber et al. 1994); however, the assumption that the mean free path is independent of distance can be questioned. For example, if we assume that the scattering mean free path scales with the radial distance, then we would infer a slightly larger average mean free path, $\sim 0.11 \mathrm{au}$, than that inferred with no radial dependence.

Our results show that compressive acceleration of seed populations requires a scattering mean free path smaller than $\sim 0.006 \mathrm{au}$, which is about 17 times smaller than the $\sim 0.1 \mathrm{au}$ mean free path estimated for energetic particle propagation from the April $21 \mathrm{CME}$ ejection. In other words, compressive acceleration of seed populations requires a reduced scattering mean free path within the compression region. It is possible that this reduced mean free path is the natural outcome of compression, or that the CME itself plays a role in reducing the scattering mean free path.

The reduced mean free path in the ICME compression is also consistent with an instability driven by the streaming and subsequent scattering of high fluxes of energetic protons (Melrose 1980; Stix 1992). The instability was invoked in models of wave growth and diffusive shock acceleration (Lee 1983). Observations show that 3-6 MeV proton intensities early in large gradual events did not exceed a plateau value of $\sim 100-200\left(\mathrm{~cm}^{2} \mathrm{sr} \mathrm{s} \mathrm{MeV}\right)^{-1}$ (Reames 1990), which was subsequently dubbed the "streaming limit," although intensities could rise much higher during passage of the shock. Observations ( $\mathrm{Ng} \&$ Reames 1994) show that wave growth greatly limits the flow and streaming of protons. Although the differential fluxes observed in this paper are significantly lower than the streaming limit, the reduced scattering mean free path within the compression appears generally consistent with streaming-limited scenarios. Observations were also used to extend the streaming limit to higher energies (Reames \& $\mathrm{Ng}$ 1998) and showed how the low-energy spectra can be flattened, but only when sufficient intensities of high-energy protons precede them (Reames \& Ng 2010). Therefore, the presence of high fluxes of seed populations preceding the events observed may be critical to limiting the scattering mean free path throughout the compression region.

Given the reduced scattering mean free path of 0.006 au in the compression region, we estimate in Appendix D that the time required for diffusive acceleration to $1 \mathrm{MeV}$ requires more than 2.5 days. This result demonstrates that even with a reduced mean free path within the compression region, the local particle acceleration rate to $1 \mathrm{MeV}$ is still too low to account for the changes in fluxes observed throughout the event. This low acceleration rate therefore reinforces the need for preexisting seed populations that are fed the compression region and demonstrates why the energetic particle spectrum within the compression region remains so similar compared to the differential energy fluxes up- and downstream from the compression.

Given the importance of preexisting seed populations needed to explain the observations within the ICME compression, it is important to ask further how these seed populations were generated. The results obtained from the changes to differential energy spectra shown in Figure 2 indicate that compression of seed populations within the solar wind plasma accounts for the increase in differential energy fluxes during the passage of the ICME. However, the question remains as to how the seed populations are produced. As discussed previously, the presence of enhanced ${ }^{3} \mathrm{He}$ throughout the events provides definitive evidence that flares contribute to the seed populations observed (Mason et al. 1986, 2002; Reames 1999; Desai et al. 2003). Curiously though, the compression ratio of $r_{c} \approx 1.7$ inferred for ICME compression would yield a differential energy spectrum with a power law of $E^{-2.6}$ based on DSA. The power law above $1 \mathrm{MeV}$ is $\sim E^{-2.8}$, similar to the DSA prediction, and we observe a steeper power law of $\sim E^{-4.2}$ above $\sim 3 \mathrm{MeV}$. This characteristic broken power-law distribution was described by Schwadron et al. (2015a, 2015b) as a product of particle acceleration driven by CMEs in the low corona. Alternatively, Li et al. (2009) demonstrated that such broken power laws may also naturally result from quasi-perpendicular shocks.

Another feature observed in the spectrum below $1 \mathrm{MeV}$ is a differential flux power law of $E^{-1.7}$. Based on the diffusion calculation presented previously, it appears that the $<1 \mathrm{MeV}$ protons are sufficiently immobile so that they cannot propagate directly from the April $21 \mathrm{CME}$ and flare source to PSP during the ICME passage. In other words, the $<1 \mathrm{MeV}$ particles are likely to interact over longer propagation periods within the solar wind plasma prior to being swept up by the ICME compression. It may not be surprising then that these particles exhibit a harder spectrum. In fact, the spectrum is so hard that it is close to the $E^{-1.5}$ limit of possible stationary-state plasma distributions out of equilibrium (Livadiotis \& McComas 2009, 2010). The seed population below $1 \mathrm{MeV}$ is likely a superposition of particles from multiple flares and compressions in the solar wind. 
Schwadron et al. (2010) argued that superposed distributions are a natural source of kappa distributions with hard suprathermal power laws in the typical range of $E^{-2.5}-E^{-1.5}$. The $E^{-1.5}$ spectrum was also considered a "ubiquitous" characteristic of the low-energy seed population (Fisk \& Gloeckler 2006) within the solar wind. A pump mechanism detailed by Fisk \& Gloeckler (2008) and Fisk et al. (2010) to account for this $E^{-1.5}$ spectrum remains controversial (Jokipii \& Lee 2010).

We conclude this section by noting the significant differences between the IS $\odot$ IS observations from 2019 April 18 to 24 compared to the observations on 2018 November 11 (Giacalone et al. 2020) of SEPs produced by a slow CME when $P S P$ was at $\sim 0.25$ au. The particle event showed a velocity dispersion with higher energy protons arriving well before the lower energy ones. After onset, the particle intensities increased gradually over a period of a few hours, reaching a peak, and then decayed gradually before the arrival of the CME at PSP. The SEP intensity decreased significantly when the CME crossed PSP. The differential energy spectrum was nearly a power law as a function of energy with a soft $E^{-4.73}$ spectrum (40-200 keV). By comparison, the spectral slope in the 2019 April 21 event below $1 \mathrm{MeV}$ was much harder, $E^{-1.7}$, but the higher energy slope above $3 \mathrm{MeV}$ was also quite soft, $E^{-4.2}$.

During the 2018 November 11 event, anisotropies show that the earliest arriving particles moved radially outward from the Sun along the interplanetary magnetic field. However, later in the event, the observed anisotropies are consistent with the advection of an isotropic distribution. This behavior is consistent with the observations throughout the 2019 April 21 event, indicating significant interplanetary scattering of the energetic particles.

\section{Summary and Conclusions}

We have investigated energetic particles observed by IS $\odot$ IS from 2019 April 18 through 20. During this period, the Sun released multiple CMEs, three of which propagated out relatively near $P S P$. This period was unique because the $P S P$ spacecraft was close to being magnetically connected to the spacecraft near Earth. The vantage point of PSP in the inner heliosphere at $\sim 0.5$ au during a period of generally low activity provided us with the ability to observe relatively isolated CME events and their interaction with energetic particle seed populations.

We observed a time period on April 21 in which the flank of an ICME passed over the PSP spacecraft. The solar wind plasma ahead of the ICME contained strong enhancements in energetic particle fluxes that appear to have been compressed and accelerated within the sheath ahead of the CME. The contemporaneous observation of strong ${ }^{3} \mathrm{He}$ enhancements (Wiedenbeck 2020) confirm that seed populations are rich in material released by solar flares. Back-mapping of the solar wind's magnetic field on April 21 place the field-line footpoints close to the boundaries of Active Region 12738. Each of the events observed on April 18, 20, and 21 were also associated with the release of CMEs from the Sun. And, on April 21, we observed a broken power law above $1 \mathrm{MeV}$ in the compressed ICME sheath consistent with predictions of diffusive shock acceleration from shocks or compressions from low in the corona.

Observations show that the passage of the ICME on April 21 was associated with an abrupt increase in energetic particle fluxes (see Figures 1 and 2). This abrupt increase is inconsistent with diffusive shock acceleration, which invokes a diffusive ramp that increases exponentially to the shock interface (Equation 3). The abrupt increase in differential fluxes is observed together with the lack of a clearly defined shock, the general presence of seed populations upstream of the ICME, and a differential energy spectrum that approximately maintains its form before, during, and after ICME passage. Together, these observations suggest that compression in front of the ICME also enhances the fluxes of energetic particle seed populations.

In Appendix D, we discuss a compression mechanism to explain the enhanced energetic particle fluxes ahead of the ICME. The mechanism has no free parameters: the compression ratio $r_{c}$ and the seed population spectral index $\gamma$ determine the enhancement in the energetic particle flux within the compression region (Equation (11)). The fluxes predicted by the mechanism are generally similar to observations within the ICME-driven compression (Figure 2).

The local enhancement of energetic particle seed populations requires restricted propagation within the compression. If this restricted propagation is caused by increased scattering, the mean free path of a $1 \mathrm{MeV}$ proton would have to be less than 0.006 au or $\sim 1.3 R_{s}$ within the compression (see Section 4).

In comparing the seed population before and after the ICME passage in Figure 2, we observe some increase in energetic particle fluxes above $1 \mathrm{MeV}$ after the ICME passage. These increased fluxes were likely associated with energetic particles and flare particles associated with the April 21 04:47 UTC CME, as detailed in Section 4. The timing of the changes in the $>1 \mathrm{MeV}$ seed population fluxes indicates a scattering mean free path for the energetic particle seed populations of $\sim 0.1$ au, more than a decade larger than the $\sim 0.006$ au mean free path needed to restrict seed populations within the ICME compression. It is possible that the restricted propagation within the compression region is the natural outcome of compressed plasma, or that the ICME itself plays a role in reducing the scattering mean free path. Wave growth may greatly limit the flow and streaming of protons (Ng \& Reames 1994), and the observed restricted propagation has physical similarities to observations of streaming-limited energetic particle fluxes (Reames 1990). Future theoretical work is needed to develop a deeper understanding of the restricted propagation within the ICME compression.

With a reduced scattering mean free path of $0.006 \mathrm{au}$ in the ICME compression region, the diffusive shock acceleration to $1 \mathrm{MeV}$ would require more than 2.5 days. It is therefore unlikely that the local enhancements observed on April 21 at PSP can be accounted for by local diffusive shock acceleration. Consistently, the observed energy spectrum within the ICME compression in Figure 2 does not show significant changes in the differential energy spectrum beyond the increased fluxes at all energies observed.

A break in the differential energy spectrum at $\sim 3 \mathrm{MeV}$ is observed throughout the observed events (see Figure 2). The compression ratio of $r_{c} \approx 1.7$ inferred for the ICME compression would yield a differential energy spectrum with a power law of $E^{-2.6}$ based on diffusive shock acceleration. The power law above $1 \mathrm{MeV}$ is $\sim E^{-2.8}$, similar to this prediction, and we observe a steeper power law of $\sim E^{-4.2}$ above $\sim 3 \mathrm{MeV}$. This characteristic broken power-law distribution was described by Schwadron et al. (2015a, 2015b) as a product of particle acceleration driven by CMEs in the low corona. Li et al. (2009) found that these broken power laws result from quasiperpendicular shocks. These particle acceleration scenarios for the seed population would require the formation of stronger shocks or compressions and much smaller scattering mean free 
paths in the strong magnetic fields low in the corona near these shocks and compressions for rapid particle acceleration.

We return to the open question as to how the energetic particle seed populations are fed into particle acceleration at interplanetary shocks. It has been widely known that energetic particle seed populations are often rich with nearly scatter-free electrons and species such as ${ }^{3} \mathrm{He}$, known to be flare associated (Mason et al. 1986, 2002; Reames 1999; Desai et al. 2003). The enhancements in energetic particle seed populations observed in this study demonstrate how the early evolution of ICMEs could enhance the fluxes of energetic particle seed populations, which precondition the particle acceleration process at distances farther from the Sun where compressions can steepen into shocks.

The IS $\odot$ IS observations below $1 \mathrm{MeV}$ show a very hard $E^{-1.7}$ energy spectrum that is likely a superposition of particles from multiple flares and compressions in the solar wind. The spectrum is so hard that it is close to the $E^{-1.5}$ limit of possible stationary-state plasma distributions out of equilibrium (Livadiotis \& McComas 2009, 2010), which suggests that suprathermal particles may play a more fundamental role for the pressure and heating of the solar wind.

The SEP acceleration process relies on solar flares to produce energetic particle seed populations and the acceleration of seed populations by compressions and shocks driven by CMEs as these structures propagate through the interplanetary medium. PSP was at the right place and at the right time to observe the compression of energetic particle seed populations. Thus, we have observed a key part of the preacceleration process that occurs close to the Sun in the development of energetic particle events. The enhancement of energetic particle seed populations observed here within the CME-driven compression could precondition the production of larger fluxes of higher energy accelerated particles as the compression region grows and steepens farther out in the heliosphere.

We are deeply indebted to everyone who helped make the Parker Solar Probe (PSP) mission possible. We thank all of the outstanding scientists, engineers, technicians, and administrative support people across all of the IS $\odot$ IS, FIELDS, and SWEAP institutions that produced and supported the IS $\odot$ IS, FIELDS, and SWEAP instrument suites, support its operations and the scientific analysis of its data. This work was supported as a part of the PSP

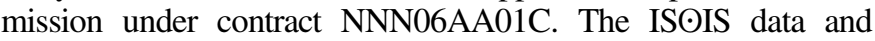
visualization tools are available to the community at: https:// spacephysics.princeton.edu/missions-instruments/isois; data are also available via the NASA Space Physics Data Facility (https:// spdf.gsfc.nasa.gov/). Parker Solar Probe was designed, built, and is now operatedby the Johns Hopkins Applied Physics Laboratory aspart of NASA's Living with a Star (LWS) program (contract NNN06AA01C). Support from the LWS management and technical team has played a critical role in the success of the Parker Solar Probe mission. We thank and acknowledge Dr. X.P. Zhao for providing the CSSS model.

\section{Appendix A Electron Observations}

EPAM observations (Gold et al. 1998) on the ACE spacecraft at the Lagrangian 1 (L1) point provide context for the observations made by PSP from 2019 April 18 through 24. We use 20 minute average and fine-time resolution measurements of the angular distribution of energetic electrons in the energy range 45-290 keV detected by the sunward-looking telescope LEFS60 of EPAM. Inspection showed that the intensity profile of the LEFS60 response tracks with the intensity profile of the magnetically deflected electrons. Thus, the LEFS60 response is primarily due to electrons. The LEFS60 telescope has a geometrical factor equal to $\sim 0.397 \mathrm{~cm}^{2} \mathrm{sr}$. The number 60 (in "LEFS60") denotes the angle that the collimator centerline of the telescope makes with the spacecraft spin axis.

The 20 minute averaged measurements of electrons (DE) measured by the B detector of the CA60 telescope are reported in the energy ranges DE1 (38-53 keV), DE2 (53-103 keV), DE3 (103-175 keV), and DE4 (175-315 keV). The CA60 has a geometrical factor of $0.103 \mathrm{~cm}^{2} \mathrm{sr}$. We also present 20 minute averaged measurements of energetic ion intensities from $A C E$ in the energy range $1.1-4.9 \mathrm{MeV}$ as detected by the LEMS120 telescope.

Figure 11 shows $80 \mathrm{~s}$ averages of the maximum intensity of the E'2 channel for the 2019 April 20 event. These data correspond to $62-102 \mathrm{keV}$ electrons measured in one of the eight sunward-looking sectors of the LEFS60 telescope. In this case, the E'2 channel is chosen for the determination of a clear onset. Even if there were a residual straggling effect in this channel, this instrumental effect leads to an underestimate of the delay for the onset of this channel. Therefore, the onset in this channel gives us (at the worst) a lower bound on the actual onset at those energies (Haggerty \& Roelof 2002). Because the energy spectrum of this particular electron event is steep, the straggling effect of higher energy channels on this channel is not expected to be significant.

Estimated instrumental background values have been subtracted from the electron intensities. The derived onset time of the electron event, based on the $2 \sigma$ data-driven onset time determination method (see Malandraki et al. 2012 for more details on this onset time determination method) is marked by the red vertical line at 01:13 UTC on 2019 April 20. Taking the FWHM of the electron pitch-angle distributions (PADs) at 01:45 UTC, an effective pitch angle of $45^{\circ}$ is obtained. The transit time for a $45^{\circ}$ pitch angle along a nominal 1.2 au long Parker spiral, for the mean energy of the E'2 channel, is $~ 29$ minutes; therefore, the anticipated electron release time at the Sun is found to be 00:44 UTC on April 20. This release time is roughly 40 minutes prior to the corresponding CME release time listed in Table 1, implying that the electron release likely occurred near the CME initiation period.

The briefest of the three events began on April 20, around 09:15 UTC during the decay phase of the event that occurred earlier (00:44 UTC) that day. This electron event did not have a clear association with an ion event, but the onset time lines up with the second type III event that occurred on April 20.

In Figure 12, we show 1 minute averages of the maximum intensity of the E'3 channel during the 2019 April 21 event. These data correspond to $102-175 \mathrm{keV}$ energetic electrons that stream away from the Sun as measured in one of the eight sunward-looking sectors of the LEFS60 telescope. In this event, the two lower energy electron channels (E'1 and E'2) have an enhanced ambient flux, due to a prior weak electron event, which masks the event onset fluxes and makes it difficult to accurately determine onset times in these channels. Furthermore, these two channels can be strongly affected by straggling of the higher energy electrons (depending on the steepness of the spectrum), whereas this effect is negligible in the two highest channels (E'3 and E'4), as previously highlighted by Haggerty \& Roelof (2002, 2003). Because for 


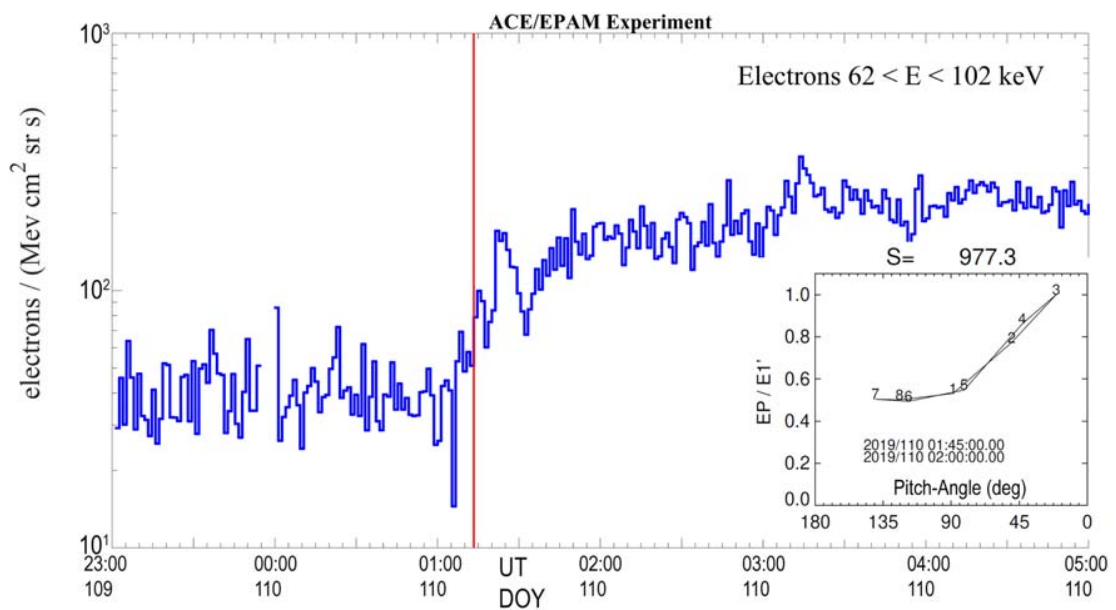

Figure 11. Observations of energetic electrons during the onset (red vertical line) of the 2019 April 20 event. The inset shows the maximum normalized intensity as a function of pitch angle for the 62-102 keV electrons streaming away from the Sun measured in one of the sectors of the LEFS60 sunward-looking telescope. This is a representative snapshot of the highly anisotropic electron pitch-angle distributions observed during this event.

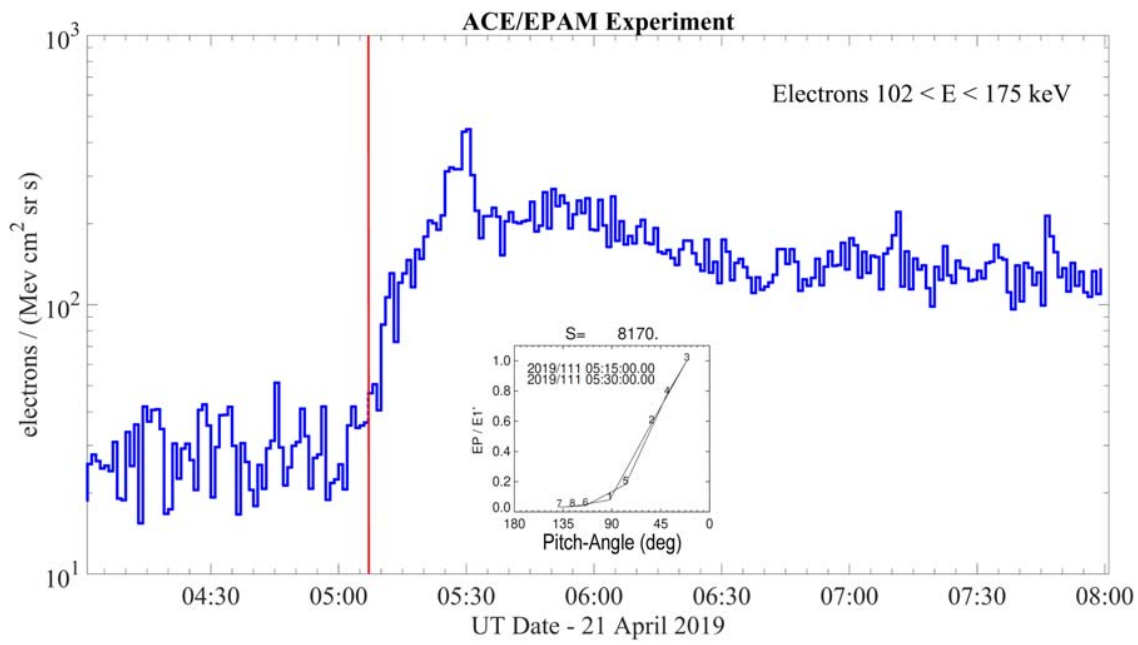

Figure 12. Observations of energetic electrons during the onset of the 2019 April 21 event. The maximum intensity of the $102-175$ keV electrons streaming away from the Sun measured in one of the sectors of the sunward-looking telescope LEFS60 for each 1 minute interval is shown. The inset presents 15 minute averaged PADs of 45-62 keV electrons at 05:15 UT, 2019 April 21, which exhibit a strong anisotropy directed parallel to the IMF. Normalized differential intensity is plotted vs. pitch angle. The red vertical line indicates the time of the determined onset of the electron event.

this electron event the E'4 electron channel enhancement was rather weak, we have utilized the E' 3 channel measurements as the highest energy channel (i.e., the highest velocity electrons) where an onset time can be reliably determined.

The red vertical line marks the time of the onset of the electron event, at 05:07 UTC, which was determined as the time that the intensity exceeded the background level by $2 \sigma$ (Malandraki et al. 2012). The inset of Figure 12 shows 15 minute averaged PADs for the $45-62 \mathrm{keV}$ electrons at 05:15 UTC on 2019 April 21. On that day, the radial component of the interplanetary magnetic field (IMF) was pointing away from the Sun. The electron population comprises an electron beam that exhibits a strong anisotropy directed parallel to the IMF and is therefore propagating away from the Sun. These peaked electron PADs argue for nearly scatter-free propagation of these particles during their outward transit from the corona to the $A C E$ spacecraft at L1. Taking the FWHM of the electron PADs at 05:15 UT on 2019 April 21, we obtain a value of $35^{\circ}$ as the effective pitch angle of this electron population. The transit time of an electron with a $35^{\circ}$ pitch angle, along a nominal 1.2 au Parker spiral length and with the mean energy of the E' 3 channel, is 20 minutes. Therefore, the deduced electron injection time at the Sun is found to be at 04:47 UTC on 2019 April 21, which is 13 minutes prior to the CME release time listed in Table 1.

\section{Appendix B CSSS Model}

We used the CSSS model (Zhao \& Hoeksema 1995; Poduval \& Zhao 2014; Poduval 2016; Jackson et al. 2019) of the corona in magnetostatic equilibrium (Bogdan \& Low 1986). The analytical solutions (Bogdan \& Low 1986) incorporate volume and sheet currents effectively (see Zhao \& Hoeksema 1995 and the references therein) by dividing the corona into three regions separated by two concentric spherical surfaces: the inner surface, the cusp surface (associated with the cusps of helmet streamers) placed at around $2.5 R_{s}$, and the outer source surface (Figure 1 in Zhao \& Hoeksema 1995) placed at $\sim 15 R_{s}$. The magnetic field lines are open at the cusp surface though still nonradial until the source surface where the solar wind moves radially out into the heliosphere. 
The CSSS model extrapolates the observed photospheric magnetic field to obtain the coronal magnetic field. The model takes the synoptic map of the photospheric magnetic field as input and employs a spherical harmonic expansion to compute the coronal magnetic field for the inner, middle, and outer coronal regions separated by the cusp and source surfaces. For the mapping presented in Figure 7, we used the synoptic map constructed using the high-resolution, high-cadence magnetograms taken by the
Helioseismic and Magnetic Imager telescope on board the Solar Dynamics Observatory for Carrington Rotation CR 2216 (2019 April 8-May 5).

\section{Appendix C \\ Enlil Modeling of the 2019 April 21 CME}

Enlil is a time-dependent 3D MHD model of the heliosphere (Odstrcil 2003). It solves equations for plasma mass, momentum
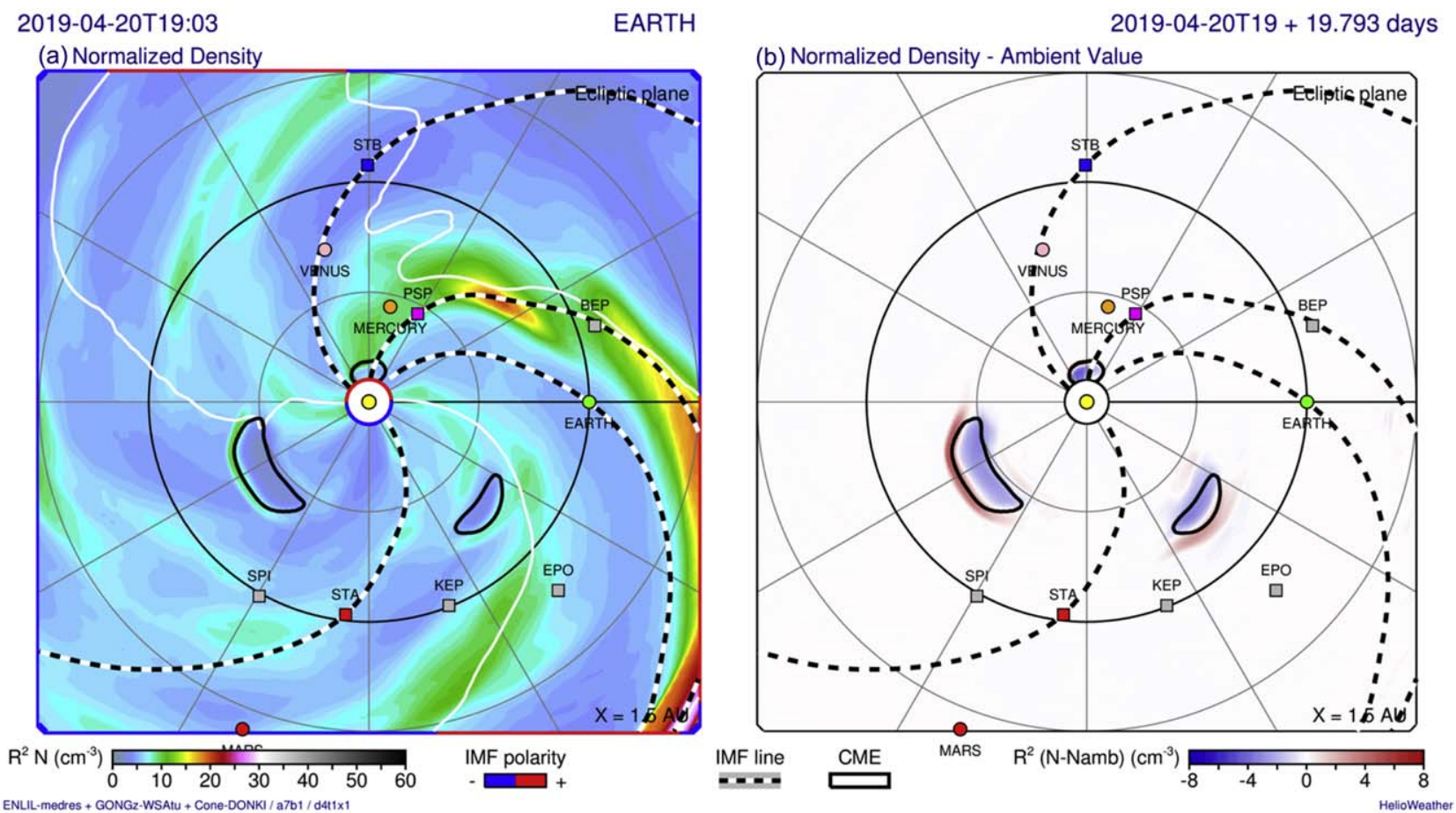

2019-04-21T16:03

EARTH

(a) Normalized Density

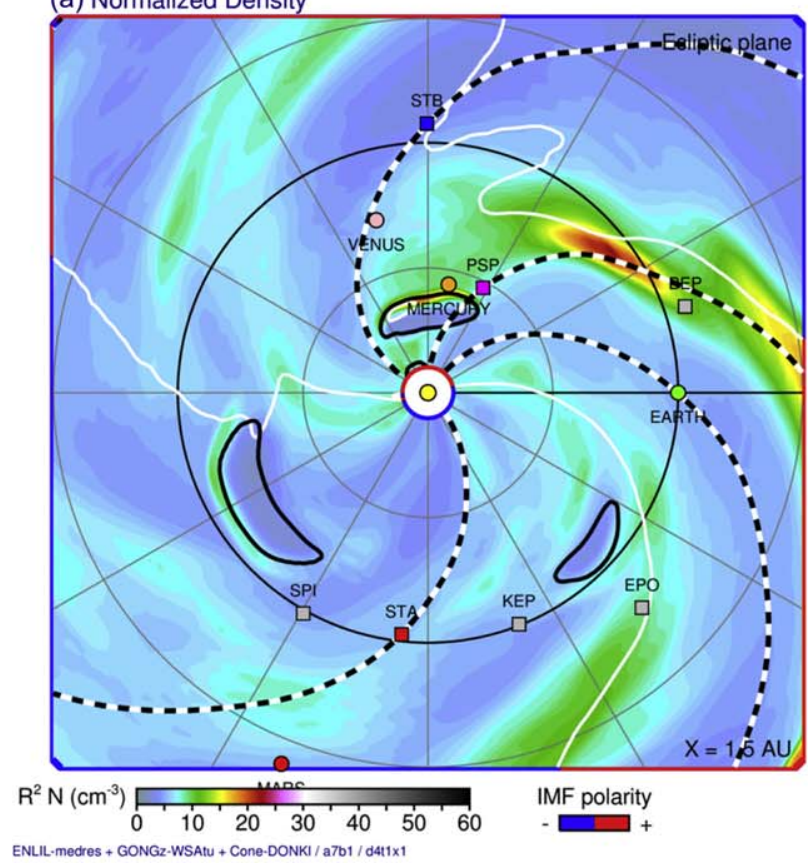

(b) Normalized Density - Ambient Value

$2019-04-21 \mathrm{~T} 16+20.668$ days

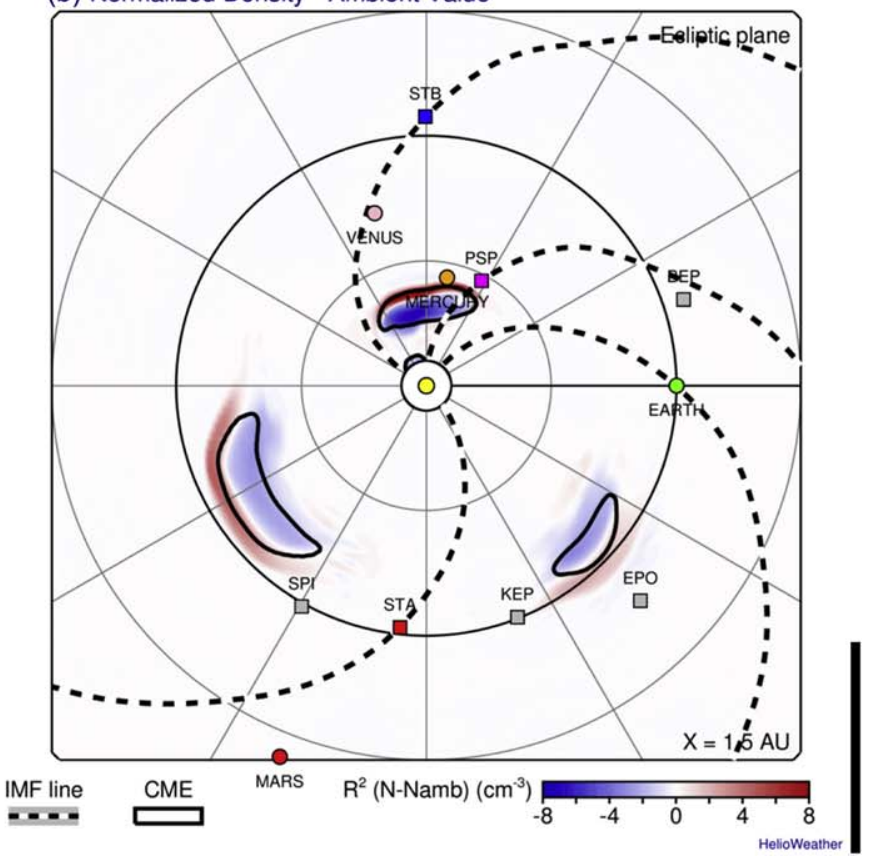

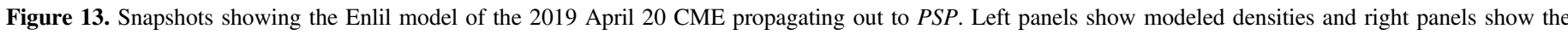

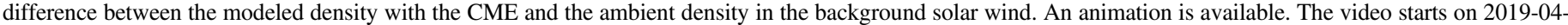
01T02:01 and ends at 2019-05-01T00:02. The real-time video duration is $29 \mathrm{~s}$.

(An animation of this figure is available.) 
2019-04-22T08:00

(a) Normalized Density
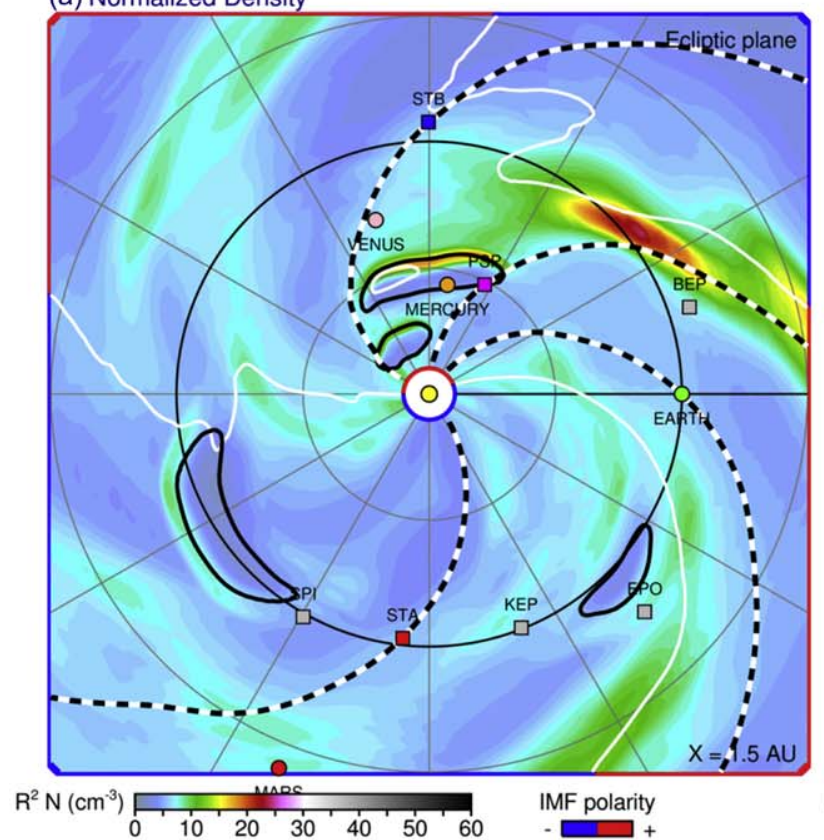

ENLLL-medres + GONGZ-WSAtu + Cone-DONK1 / a7b1 / d4t1 1 1

2019-04-23T22:02

(a) Normalized Density

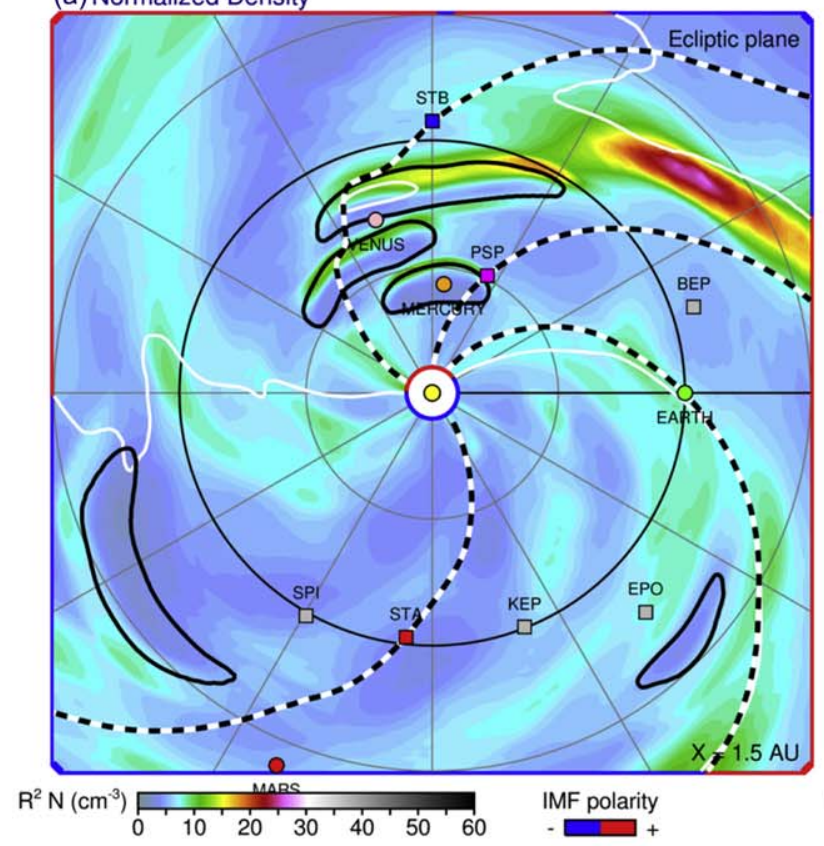

EARTH (b) Normalized Density - Ambient Value

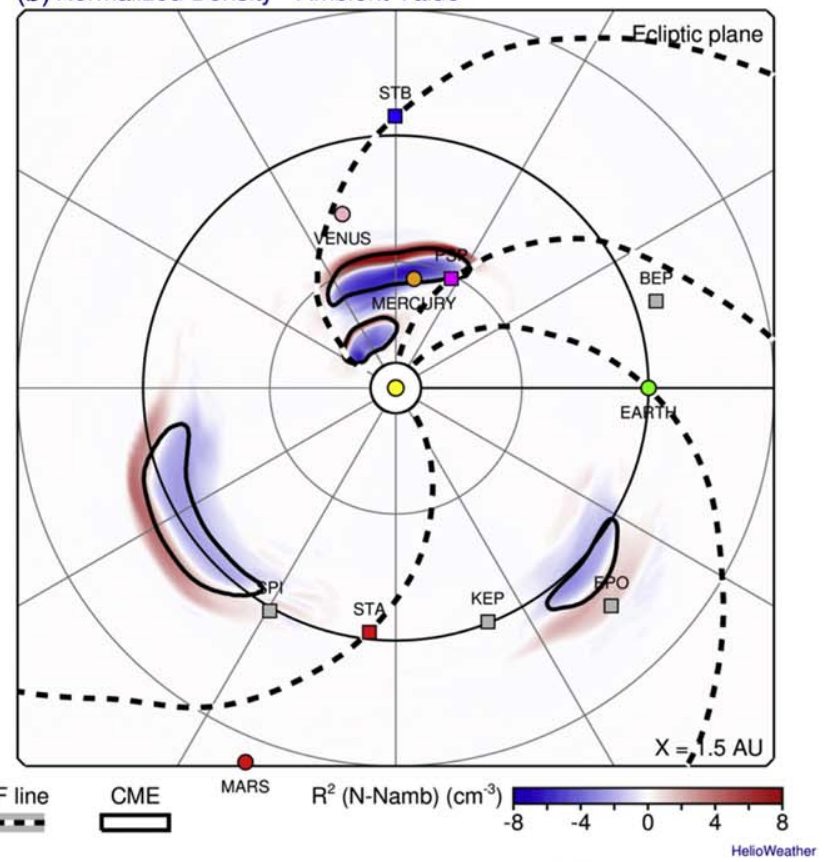

2019-04-23T22 + 22.918 days

(b) Normalized Density - Ambient Value

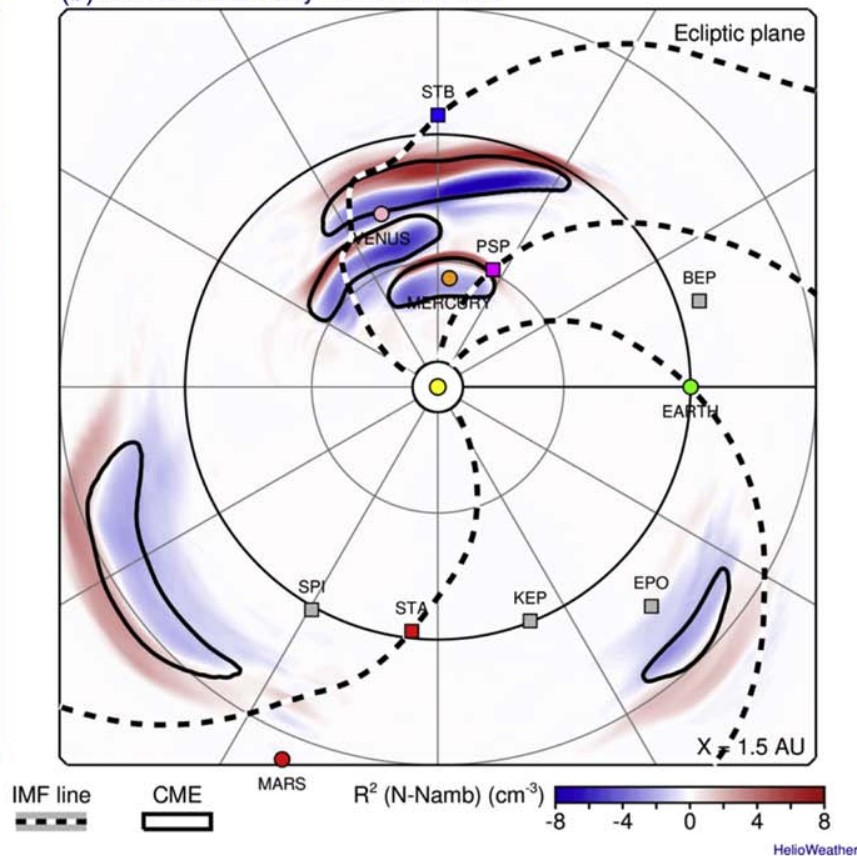

Figure 14. Enlil model snapshots similar to Figure 13 showing the stages of the CME's propagation after April 21 out to 1 au.

and energy density, and magnetic field, using a flux-corrected transport algorithm. The Enlil cone model forecasts CME propagation from the Enlil inner boundary (at $21.5 R_{s}$ ) to 2 au. The ambient solar wind is based on the WSA model (Arge \& Pizzo 2000). In the Enlil cone model, the CME propagates out close to the Sun with constant angular and radial velocity. The Enlil model takes the following input parameters at its inner boundary.

1. Start time at $21.5 R_{s}: 2019$ April 20 at 10:33 UTC.

2. Direction: HEEQ longitude $90^{\circ}$ and latitude $2^{\circ}$.
3. Half angular width: $30^{\circ}$ half of the full angular width of the cone.

4. Speed: $387 \mathrm{~km} \mathrm{~s}^{-1}$ radial velocity $\left(\mathrm{km} \mathrm{s}^{-1}\right)$ at the Enlil inner boundary.

Figures 13 and 14 show the evolution of the CME released on April 20. The structure propagates to and beyond PSP, driving a large compression in front of the structure. These images are taken from a movie available with Figure 13. Figure 15 shows the timeline of the modeled plasma at the location of the PSP spacecraft. 


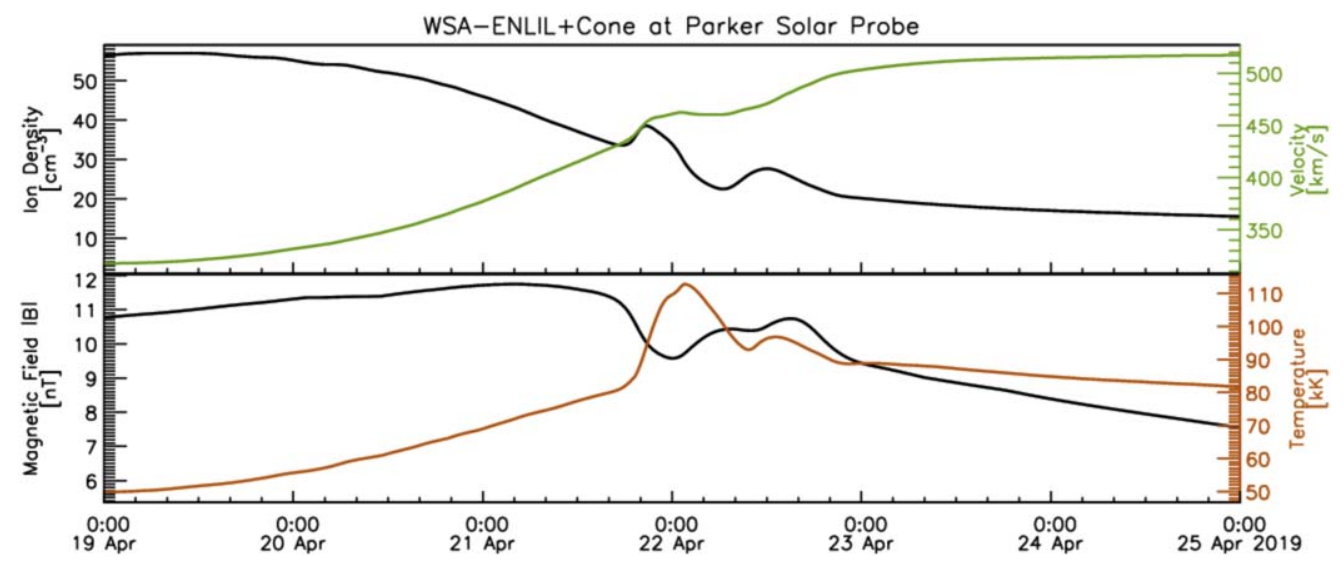

Figure 15. Timeline of the Enlil model results at the location of the PSP spacecraft: (top panel) ion density (black), solar wind and CME velocity (green); (bottom panel) magnetic field strength (black), and plasma temperature (orange).

\section{Appendix D}

\section{Compressive Enhancement of Energetic Particles}

In this appendix, we describe a scenario in which energetic particles are compressed by the plasma as they move into a compression region, as illustrated in Figure 16. Our treatment departs from the conventional solution for DSA. We begin by describing how diffusive ramps are treated within DSA theory and show how compressive enhancements differ.

For simplicity, we take the compression formed simply from faster solar wind plasma with speed $u_{f}$ ramming into a slower plasma with speed $u_{s}$. The compression ratio is $r_{c}=u_{f} / u_{s}$ and the local width of the compression region with a speed gradient is taken to be $\delta x$. We assume that the magnetic field and the solar wind direction are aligned in the $x$-direction.

The evolution of the isotropic part of the distribution function is typically described using the Parker transport equation (Parker 1965):

$$
\begin{aligned}
\frac{\partial f_{0}}{\partial t} & +\boldsymbol{u} \cdot \nabla f_{0}-\nabla \cdot\left(\boldsymbol{K} \cdot \nabla f_{0}\right) \\
& -\frac{\nabla \cdot \boldsymbol{u}}{3} p \frac{\partial f_{0}}{\partial p}=0,
\end{aligned}
$$

where $\boldsymbol{u}$ is the solar wind velocity and $\boldsymbol{K}$ is the diffusion tensor. In DSA theory, a discontinuity between the fast and slow wind creates the conditions for rapid particle acceleration. Upstream from the fast-slow wind interface, there is a balance of diffusive streaming against the convected fast solar wind,

$$
u_{f} f_{0}-\kappa \frac{\partial f_{0}}{\partial x}=0 .
$$

The convective-diffusion solution follows,

$$
f_{u}(x)=f_{s} \exp \left(x u_{f} / \kappa\right),
$$

where $f_{s}$ is the isotropic part of the distribution at the stream interface, $x=0$, and $f_{u}$ is the upstream solution. The upstream solution in Equation (3) results from the outward convection of energetic particles away from the Sun and diffusive streaming back toward the Sun. It is important to note, however, that we have assumed the source is a delta function at $x=0$. If there is a seed population, then the correct upstream solution involves both the shock-accelerated population that streams against the flow and the preexisting seed population that convects with the flow.

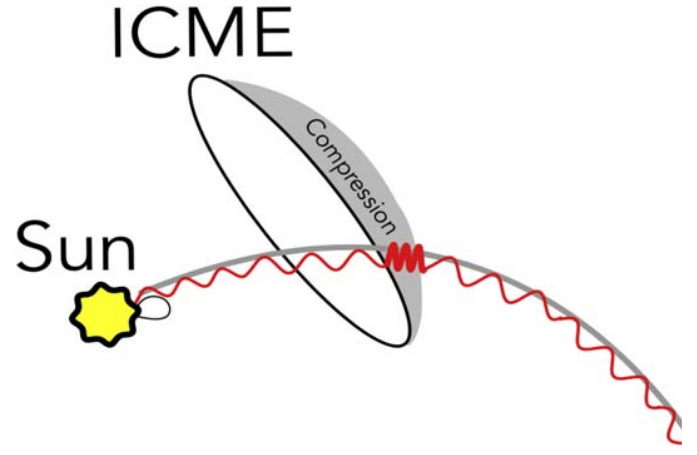

Figure 16. Illustration of a CME-driven compression region including energetic particles that diffuse away from the Sun after a flare or particle acceleration from the low corona.

We consider a different scenario in which the gradient in the solar wind speed is not a discontinuity. We take the distribution to remain approximately isotropic as it is convected into the speed gradient such that the rate of convection exceeds the rate of diffusion. In this departure from DSA theory, we take

$$
u \frac{\partial f_{0}}{\partial x}>\frac{\partial}{\partial x}\left(\kappa \frac{\partial f_{0}}{\partial x}\right)
$$

This requires that $u>\kappa / \delta x$. Equivalently, this places a requirement on the scattering mean free path,

$$
\lambda<3 u \delta x / v \text {. }
$$

Taking $u=400 \mathrm{~km} \mathrm{~s}^{-1}$, a width of $\delta x=0.1 \mathrm{au}$, and a $1 \mathrm{MeV}$ proton, this would require a scattering mean free path $\lambda<2 R_{s}$ (or $0.009 \mathrm{au}$ ) within the compression.

To help conceptualize the limit on the mean free path, we consider the rate of particle acceleration if the compression were instead a discontinuity. The DSA acceleration time to a given momentum $p$ is (Jokipii 1982, 1987; Drury 1983; Forman \& Drury 1983; Schwadron et al. 2015b)

$$
\tau_{p} \approx \frac{3 \delta x_{\mathrm{dsa}}}{\Delta u},
$$

where $\Delta u=u_{f}-u_{s}$, the width of the DSA acceleration region is

$$
\delta x_{\mathrm{dsa}}=\frac{\kappa_{f}}{u_{f}}+\frac{\kappa_{s}}{u_{s}}
$$


and $\kappa_{s}$ and $\kappa_{f}$ are the diffusion coefficients on the slow and fast wind sides of the stream discontinuity. For simplicity, we take $\kappa_{f}>\kappa_{s} \approx 0$, due to the presence of downstream turbulence. Using the previously stated limit for $\lambda$, Equation (5), we find that

$$
\delta x_{\mathrm{dsa}}<\delta x
$$

Not surprisingly, this implies that the region of diffusive acceleration must be smaller than the width of the compressive gradient. Conversely, if the width of the speed gradient exceeds the width of the DSA acceleration region, then compressive acceleration will dominate.

We find the DSA acceleration time by substituting Equation (8) into Equation (6),

$$
\tau_{p}<3 \frac{\delta x}{\Delta u} .
$$

The quantity $\delta x / \Delta u$ represents the convection time through the speed gradient. Therefore, the condition in Equation (9) implies that if the convection time exceeds the DSA acceleration time, then compressive acceleration will dominate.

Given the condition on the scattering mean free path in Equation (5), we find the following approximation for transport into the compression,

$$
u \frac{\partial f_{0}}{\partial x}-\frac{1}{3} \frac{\partial u}{\partial x} p \frac{\partial f_{0}}{\partial p}=0
$$

This is solved for the compressed isotropic portion of the distribution function, $f_{c}$, as a function of the distribution function $\tilde{f}_{0}$ convected into the compression,

$$
f_{c}=r_{c}^{\gamma / 3} \tilde{f}_{0} .
$$

Here, $\gamma$ is the power-law index of the energetic particle distribution in the faster wind behind the compression, $\tilde{f}_{0} \propto p^{-\gamma}$.

\section{ORCID iDs}

N. A. Schwadron (1) https://orcid.org/0000-0002-3737-9283

S. Bale (ii) https://orcid.org/0000-0002-1989-3596

A. Case (i) https://orcid.org/0000-0002-3520-4041

E. R. Christian (1) https://orcid.org/0000-0003-2134-3937

A. C. Cummings $\odot$ ic https://orcid.org/0000-0002-3840-7696

T. Dudok de Wit (1) https://orcid.org/0000-0002-4401-0943

M. I. Desai (1) https://orcid.org/0000-0002-7318-6008

C. J. Joyce (1) https://orcid.org/0000-0002-3841-5020

B. Heber (1) https://orcid.org/0000-0003-0960-5658

M. E. Hill (1) https://orcid.org/0000-0002-5674-4936

J. C. Kasper (1) https://orcid.org/0000-0002-7077-930X

K. Korreck (ib https://orcid.org/0000-0001-6095-2490

R. A. Leske (1) https://orcid.org/0000-0002-0156-2414

R. MacDowall (ㅇ) https://orcid.org/0000-0003-3112-4201

D. Malaspina (i) https://orcid.org/0000-0003-1191-1558

W. H. Matthaeus (1) https://orcid.org/0000-0001-7224-6024

D. J. McComas (1) https://orcid.org/0000-0001-6160-1158

D. G. Mitchell @ $\odot$ https://orcid.org/0000-0003-1960-2119

M. Pulupa (i) https://orcid.org/0000-0002-1573-7457

J. S. Rankin (1) https://orcid.org/0000-0002-8111-1444

M. Stevens (1) https://orcid.org/0000-0002-7728-0085

J. R. Szalay (1) https://orcid.org/0000-0003-2685-9801

R. Winslow (1) https://orcid.org/0000-0002-9276-9487

\section{References}

Arge, C. N., \& Pizzo, V. J. 2000, JGR, 105, 10465

Bale, S. D., Goetz, K., Harvey, P. R., et al. 2016, SSRv, 204, 49

Bieber, J. W., Matthaeus, W. H., \& Smith, C. W. 1994, ApJ, 420, 294

Bogdan, T., \& Low, B. 1986, ApJ, 306, 271

Bučík, R., Innes, D. E., Guo, L., Mason, G. M., \& Wiedenbeck, M. E. 2015, ApJ, 812, 53

Bučík, R., Innes, D. E., Mason, G. M., \& Wiedenbeck, M. E. 2016, ApJ, 833,63

Cane, H. V., Erickson, W. C., \& Prestage, N. P. 2002, JGRA, 107, 14

Desai, M. I., Mason, G. M., Dwyer, J. R., et al. 2003, ApJ, 588, 1149

Drury, L. O. 1983, RPPh, 46, 973

Fisk, L. A., \& Gloeckler, G. 2006, ApJL, 640, L79

Fisk, L. A., \& Gloeckler, G. 2008, ApJ, 686, 1466

Fisk, L. A., Gloeckler, G., \& Schwadron, N. A. 2010, ApJ, 720, 533

Forman, M. A., \& Drury, L. O. 1983, Proc. ICRC, 2, 267

Forman, M. A., \& Gleeson, L. J. 1975, Ap\&SS, 32, 77

Fox, N. J., Velli, M. C., Bale, S. D., et al. 2016, SSRv, 204, 7

Fränz, M., \& Harper, D. 2002, P\&SS, 50, 217

Giacalone, J., Mitchell, D. G., Allen, R. C., et al. 2020, ApJS, doi:10.3847/ $1538-4365 / a b 5221$

Gold, R., Krimigis, S., Hawkins, S., et al. 1998, SSRv, 86, 541

Gopalswamy, N., \& Mäkelä, P. 2010, ApJL, 721, L62

Haggerty, D. K., \& Roelof, E. C. 2002, ApJ, 579, 841

Haggerty, D. K., \& Roelof, E. C. 2003, AdSpR, 32, 423

Hapgood, M. A. 1992, P\&SS, 40, 711

Jackson, B., Yu, H.-S., Buffington, A., et al. 2019, SpWea, 17, 639

Jokipii, J. R. 1982, ApJ, 255, 716

Jokipii, J. R. 1987, ApJ, 313, 842

Jokipii, J. R., \& Lee, M. A. 2010, ApJ, 713, 475

Kasper, J. C., Abiad, R., Austin, G., et al. 2016, SSRv, 204, 131

Lee, M. A. 1983, JGR, 88, 6109

Li, G., Zank, G. P., Verkhoglyadova, O., et al. 2009, ApJ, 702, 998

Lin, R. P. 1970, SoPh, 12, 266

Lin, R. P. 1974, SSRv, 16, 189

Livadiotis, G., \& McComas, D. J. 2009, JGRA, 114, 11105

Livadiotis, G., \& McComas, D. J. 2010, ApJ, 714, 971

MacDowall, R. J., Lara, A., Manoharan, P. K., et al. 2003, GeoRL, 30, 8018

Malandraki, O. E., Agueda, N., Papaioannou, A., et al. 2012, SoPh, 281, 333

Malandraki, O. E., Sarris, E. T., Lanzerotti, L. J., et al. 2002, JASTP, 64, 517

Mason, G. M., Reames, D. V., Klecker, B., \& Hovestadt, D. 1986, ApJ, 303,849

Mason, G. M., Wiedenbeck, M. E., Miller, J. A., et al. 2002, ApJ, 574, 1039

McComas, D., Christian, E., Cohen, C., et al. 2019, Natur, 576, 223

McComas, D. J., Alexander, N., Angold, N., et al. 2016, SSRv, 204, 187

Melrose, D. B. 1980, Plasma Astrophysics, Vol. 1 (New York: Gordon and Breach)

Ng, C. K., \& Reames, D. V. 1994, ApJ, 424, 1032

Odstrcil, D. 2003, AdSpR, 32, 497

Ontiveros, V., \& Vourlidas, A. 2009, ApJ, 693, 267

Parker, E. N. 1965, P\&SS, 13, 9

Poduval, B. 2016, ApJL, 827, L6

Poduval, B., \& Zhao, X.-P. 2014, ApJL, 782, L22

Reames, D. V. 1990, ApJL, 358, L63

Reames, D. V. 1999, SSRv, 90, 413

Reames, D. V., \& Ng, C. K. 1998, ApJ, 504, 1002

Reames, D. V., \& Ng, C. K. 2010, ApJ, 723, 1286

Russell, C. T. 1971, CosEl, 2, 184

Schwadron, N. A., Dayeh, M. A., Desai, M., et al. 2010, ApJ, 713, 1386

Schwadron, N. A., \& Gombosi, T. I. 1994, JGR, 99, 19301

Schwadron, N. A., Lee, M. A., Gorby, M., et al. 2015a, J. Phys. Conf. Ser., 642, 012025

Schwadron, N. A., Lee, M. A., Gorby, M., et al. 2015b, ApJ, 810, 97

Stix, T. H. 1992, Waves in Plasmas (New York: AIP)

Vourlidas, A., Howard, R. A., Plunkett, S. P., et al. 2016, SSRv, 204, 83

Vourlidas, A., \& Ontiveros, V. 2009, in AIP Conf. Proc. 1183, Shock Waves in Space and Astrophysical Environments, ed. A. Xianzhi \& G. Z. Ross (Melville, NY: AIP), 139

Vourlidas, A., Wu, S. T., Wang, A. H., Subramanian, P., \& Howard, R. A. 2003, ApJ, 598, 1392

Webb, D. F., \& Howard, T. A. 2012, LRSP, 9, 3

Wiedenbeck, M., Bučík, R., Mason, G. M., et al. 2020, ApJS, doi:10.3847/ $1538-4365 / a b 5963$

Zhao, X. P., \& Hoeksema, J. T. 1995, JGR, 100, 19 